\title{
A Brief Review on Aerodynamic Performance of Wingtip Slots and Research Prospect
}

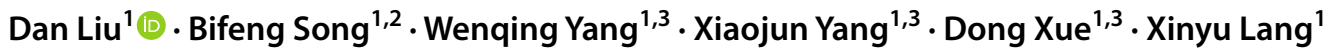

Received: 18 April 2021 / Revised: 30 August 2021 / Accepted: 8 September 2021 / Published online: 8 December 2021

(c) The Author(s) 2021

\begin{abstract}
Wingtip slots, where the outer primary feathers of birds split and spread vertically, are regarded as an evolved favorable feature that could effectively improve their aerodynamic performance. They have inspired many to perform experiments and simulations as well as to relate their results to aircraft design. This paper aims to provide guidance for the research on the aerodynamic mechanism of wingtip slots. Following a review of previous wingtip slot research, four imperfections are put forward: vacancies in research content, inconsistencies in research conclusions, limitations of early research methods, and shortage of the aerodynamic mechanism analysis. On this basis, further explorations and expansion of the influence factors for steady state are needed; more attention should be poured into the application of flow field integration method to decompose drag, and evaluation of variation in induced drag seems a more rational choice. Geometric and kinematic parameters of wingtip slot structure in the unsteady state, as well as the flexibility of wingtips, should be taken into account. As for the aerodynamic mechanism of wingtip slots, the emphasis can be placed on the study of the formation, development, and evolution of wingtip vortices on slotted wings. Besides, some research strategies and feasibility analyses are proposed for each part of the research.
\end{abstract}

Keywords Slotted wingtip $\cdot$ Tip vortex $\cdot$ Drag reduction $\cdot$ Unsteady state $\cdot$ Flexibility

\section{Introduction}

In a long-term natural evolution, birds have gradually formed wing shapes and bone structures suitable for efficient flight [1-3]. In contrast to modern aircraft, birds can appropriately change wing shape and flapping patterns in a variety of complex ways to achieve mission adaptability during flight $[4,5]$. A schematic drawing of the skeleton anatomy and feather distribution of typical bird wings is displayed in Fig. 1. The large and pointed primary feathers dispersedly arranged on the wingtip, forming the outer wing,

Bifeng Song

sbf@nwpu.edu.cn

1 School of Aeronautics, Northwestern Polytechnical University, Xi' an 710072, China

2 Research \& Development Institute of Northwestern Polytechnical University in Shenzhen, Shenzhen 518057, China

3 Yangtze River Delta Research Institute of Northwestern Polytechnical University, Taicang 215400, China acts as a crucial multifunctional aerodynamic part in bird flight [6]. This will be the research object of this paper, as later discussed in detail.

The wing planforms of birds' wings can be systematically divided into four categories (Fig. 2): (a) elliptical wing, allowing for fast and tightly controlled flight; (b) high-speed wing, adapted to sustained high-speed flight; (c) high-aspect ratio wing, excellent for soaring (flying without flapping); and (d) slotted high-lift wing, skilled in high maneuverability and heavy loading [8]. Additionally, here are some typical birds for these wing planforms listed in Table 1 .

It is worth noticing that slotted high-lift wings have greater agility than other wings. In particular, they have similar requirements as wings used by modern aircraft owing to the payload demands $[10,11]$. One notable feature of slotted high-lift wings is the presence of wingtip slots, as shown in Fig. 3. Although they vary for different bird species, usually they have prominent and separated feathers at wingtip called wingtip slots (the first 5-6 primary feathers have conspicuous emargination) [12].

The slotted wingtip, spreading out horizontally and vertically, allows the primary feathers to act as individual 


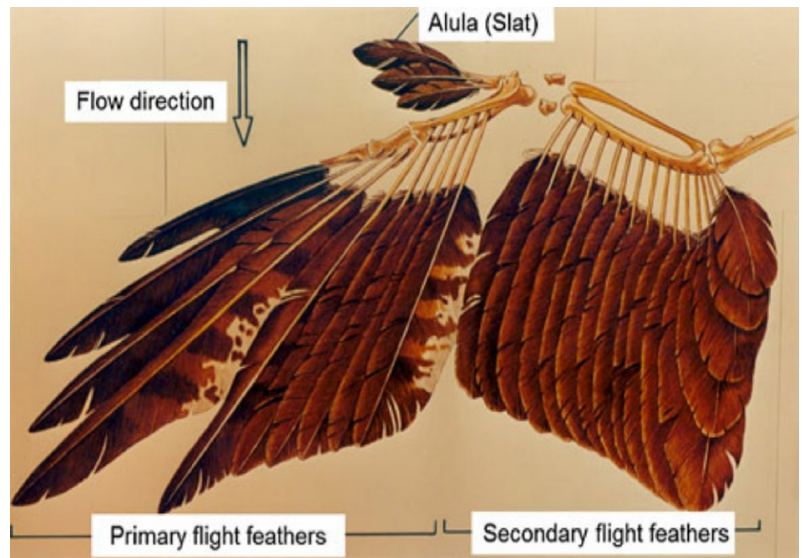

Fig. 1 Schematic drawing showing the skeleton anatomy and the feather arrangement on a typical bird wing [7]

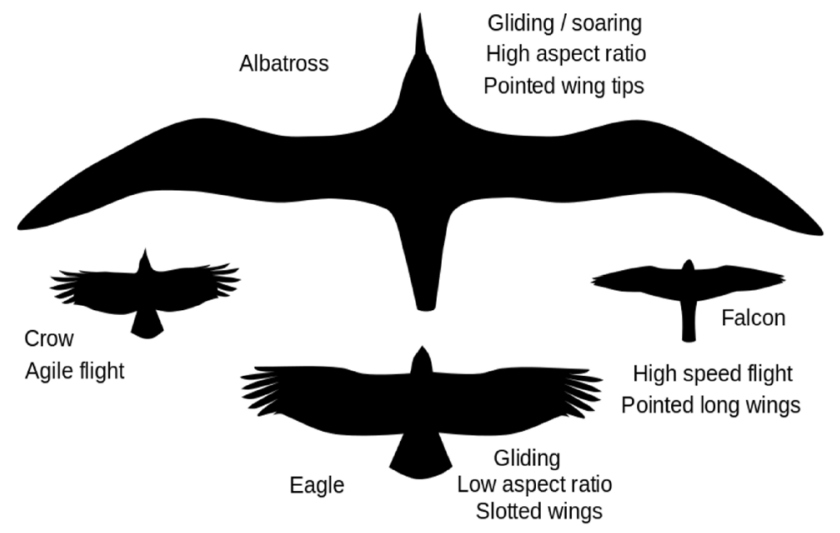

Fig. 2 Classification of wing shape: elliptical wings, high-speed wings, high-aspect ratio wings, and slotted high-lift wing [9]

aerodynamic surfaces [13]. It can break up the wingtip vortexes, which is considered as a feature of improving flight performance after the evolution of birds [14, 15].

The goal of this paper is to review the previous studies of wingtip slots and put forward some valuable issues for further research. Section 2 tries to review and summarize the studies of wingtip slots that have been carried out, and some existing problems are analyzed. Section 3 aims to offer some directions for future research based on existing imperfections and vacancies in wingtip slots' study, as well as comprehensively and systematically analyzes the feasibility of suggested research strategies.

\section{Review on Wingtip Slot Research}

\subsection{Background and Previous Works}

\subsubsection{Experiments on Biological Specimens of Bird Wings}

Withers [17] compared the aerodynamic behavior of a slotted hawk wing and a single vulture feather (Fig. 4). Figure 5 demonstrates that slotted wingtip slightly decreased drag and increased lift, significantly increased yaw moment. He proposed that wingtip had evolved due to improving biomechanical limitations to the bending strength and reducing the propensity for wingtip stall.

Tuker [18] experimented on a Harris hawk glided freely inside a wind tunnel with clipped and unclipped wingtips. The curves in Fig. 6 showed that the total drag was related to gliding speed and the number of wingtip slots. On the whole, the wings' drag of the Harris Hawk with wingtips was smaller than that without wingtips. It was found that the bird with slotted (unclipped) wingtips had a drag reduction of about $70-90 \%$ in contrast to the clipped one. The induced drag factor of unclipped feathers is about 0.56 and increased to 1.1 in clipped feathers. Thus, it has been verified that bird wingtip feathers cause a reduction in drag, as depicted in Fig. 7.

To quantify the aerodynamic properties of the avian wing, March [19] conducted wind tunnel tests with deceased biological wing specimens. Flow visualization around the slotted wingtips of a bird reveals smooth streamlines near the primary feathers (Fig. 8). The results revealed that slotted wingtips can decompose the upwash generated by the wingtip vortex and obtain extra thrust.

Kleinherenbrink et al. [20] utilized PIV (particle image velocimetry) to measures the airflow and wake around the wingtip of a jackdaw flying unconstrained in a wind tunnel. From Fig. 9, it follows that the resulting multi-cored wingtip vortex confirmed that slotted wingtips enable vorticity to spread in both gliding and flapping flight. They proposed the hypothesis that slotted wings originally evolved to enhance the performance of powered flight [21].
Table 1 Examples of representative birds for proposed wing planform classification

\begin{tabular}{ll}
\hline Wing planform & Typical figures \\
\hline Elliptical wing & Peckers, hornbills, toucans, grouses and woodcocks \\
High-speed wing & Falcons, plovers, swifts, hummingbirds, swallows, and jaegers \\
High-aspect ratio wing & Albatrosses, frigate-birds, gannets and gulls \\
Slotted high-lift wing & Vultures, eagles, ospreys, owls, harriers, raven, and pelicans \\
\hline
\end{tabular}


Fig. 3 Birds' wingtip feathers. a Kea, b Pacific Brown Pelican, c Red Tailed Hawk, d Bald Eagle, e Northern Hawk Owl, f Great Blue Heron [16]
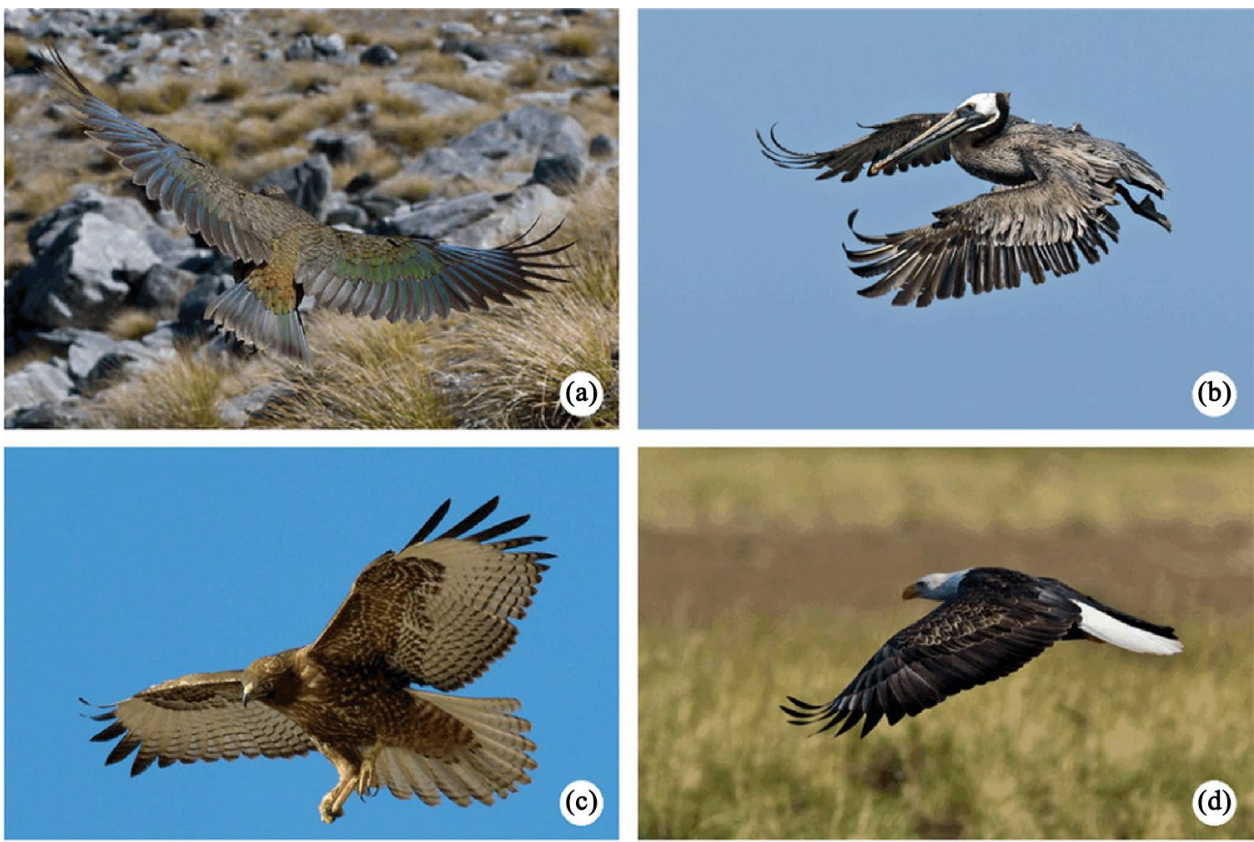

(e)

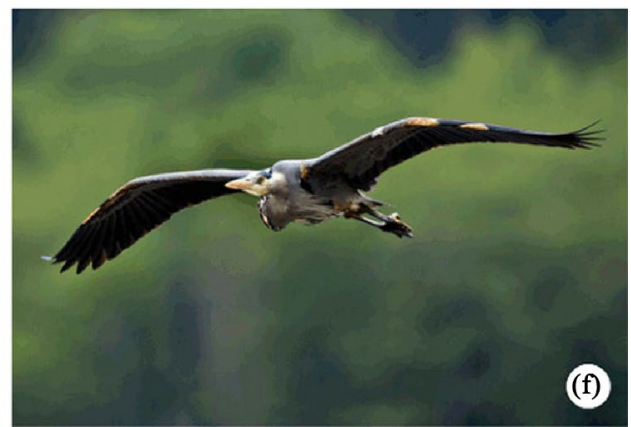

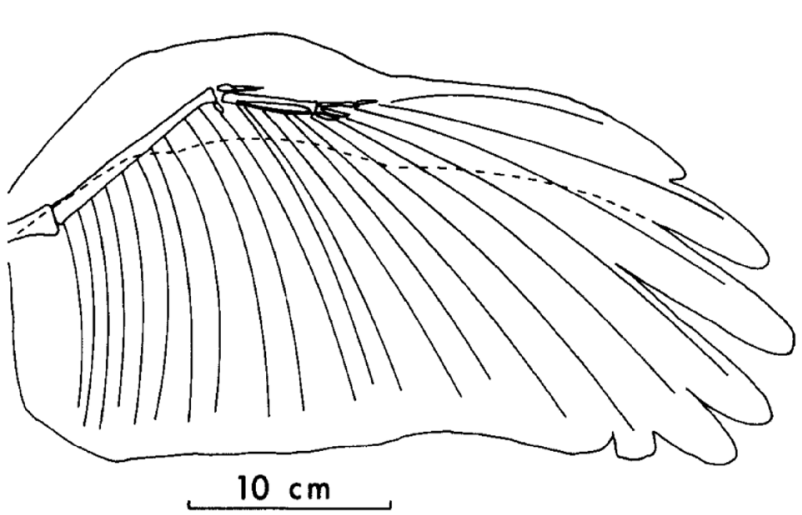

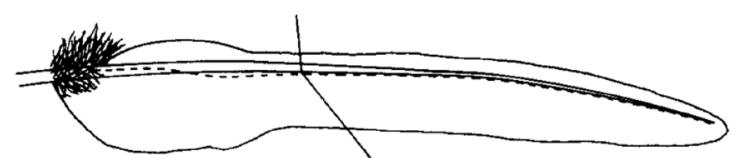

$10 \mathrm{~cm}$

Fig. 4 The planform of the Red-shouldered Hawk wing and a Black Vulture primary feather [17]

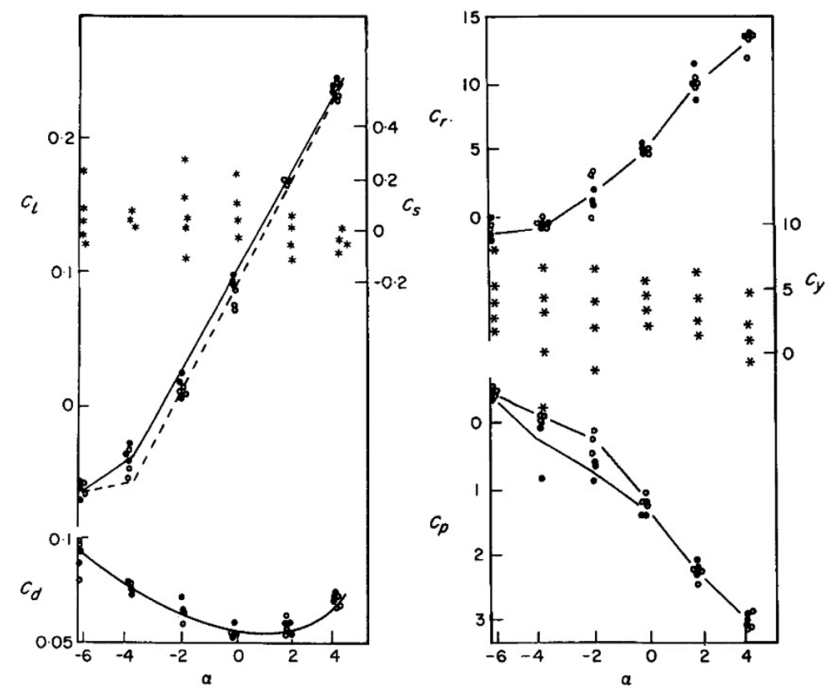

Fig. 5 Aerodynamic forces and moments for a Red-shouldered Hawk wing (lift coefficient $C_{l}$; drag coefficient $C_{d}$; side force coefficient $C_{s}$; roll moment $C_{r}$; pitching moment $C_{p}$; yaw moment $C_{y}$ ) as a function of angle of attack [17] 


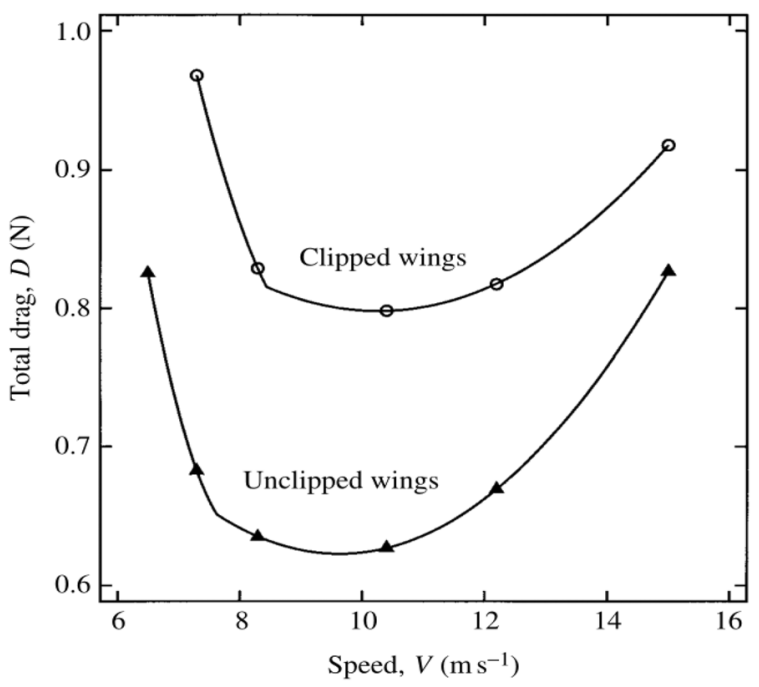

Fig. 6 Minimum total drag of the Harris' hawk at different speeds with clipped and unclipped wings [18]

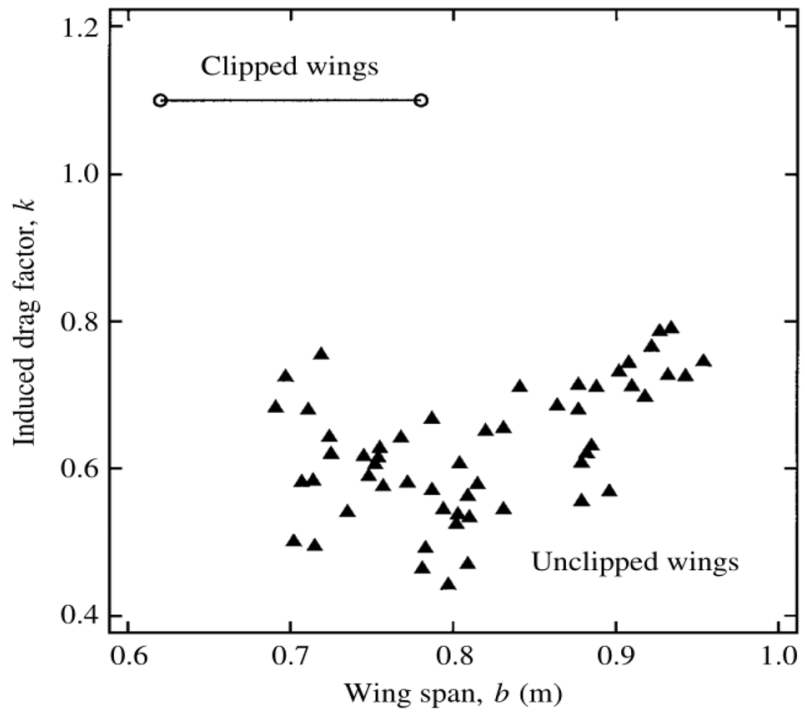

Fig. 7 The induced drag factors for the clipped and unclipped Harris' hawk at different wing spans [18]

Nadal et al. [22] proved the vital role of wingtip slots from the perspective of biostatistics. He collected feather information of 13,814 red-legged partridges to explore the relationship between the feather proportions and morphological properties of distinct ages and sexes and their rapid take-off ability: thrust is greatly formed by the distal parts of the wings.

Another interesting note was the variation in slotted wingtips' upstroke kinematics: flexed and tip-reversal. This dynamic flapping process attracts the focus of some scholars. Crandell and Tobalske [23] came to the conclusion through a flapping test with dry rock dove wings: the slotted wing generated significant aerodynamic forces during the upstroke, where drag force served as weight or thrust support. The force created during the downstroke is $115 \%$ of the body weight, and the force (thrust) produced for upstroke is $36 \%$ of the weight (Fig. 10).

\subsubsection{Numerical Simulations on Bionic Slotted Wings}

To further understand the influence wingtip slots have on bird flight characteristics, Sachs and Moelyadi ${ }^{[24]}$ analyzed the application of sweep wingtip to the slotted wing (Fig. 11a). They exhibited that wingtip slots with sweep yield a stabilizing yawing moment of significant magnitude, as illustrated in Fig. 11b, substantially growing with the lift coefficient. The demonstration was keyed to the nature of the sweep at the slotted wingtip.

Saiteja and Suresh [25] investigated the aerodynamic performance of two wings: one with wingtip slots and the other without. The results are presented in Fig. 12, which demonstrated that there was a decrease in lift coefficient and an increase in the lift-to-drag ratio for the slotted wing as compare with the base wing.

Fluck and Crawford [26] created an extended lifting line model considering the viscous drag effect to calculate the lift distribution, and drag of wings varying with cant angles, tip twist angle and number of wingtip slots, (Fig. 13). From Fig. 14, it followed that wing stall can be alleviated by increasing twist angle of the slotted wingtip. The best performance was generally found when spreading more feathers over larger cant angles out of the wing plane, as shown in Fig. 15. However, it was not found that a certain parameter can increase lift and the lift-to-drag ratio at the same time.

\subsubsection{Wind Tunnel Experiments on Bionic Slotted Wings}

Tucker [27] conducted wind tunnel experiments on wings with three types of wingtips: feathers of Harris' Hawk, slotted tip made of balsa wood and shaped as Clark Y airfoils, and unslotted Clark $\mathrm{Y}$ wingtip mounted on a base wing (Fig. 16). In Fig. 17, at an angle of attack of $10.5^{\circ}$, the wing with the slotted tip increased the lift-to-drag ratio from 4.9 to 10.1 , and the total drag reduction was found to be $12 \%$ compared to the conventional wing.

To understand the role wingtip slots with cant angles play on wings' aerodynamic behavior, Hossain et al. [28] carried out a series of experiments on slotted wings varying with cant angles (Fig. 18). The evaluation confirmed that increasing cant angles of slotted winglets can obtain lift raise and drag reduction. As depicted in Fig. 19, for the angle of attack of $8^{\circ}$, the experimental result showed slotted wing acquired $25-30 \%$ reduction in drag coefficient and a 10-20\% increase in lift. 


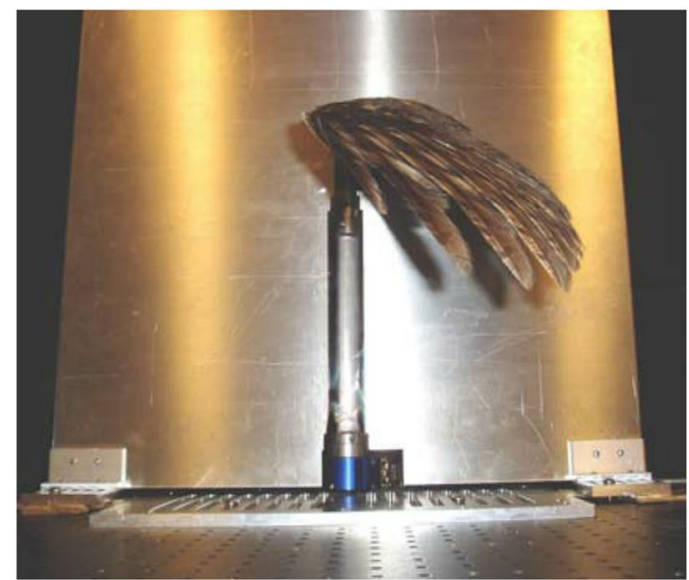

(a)

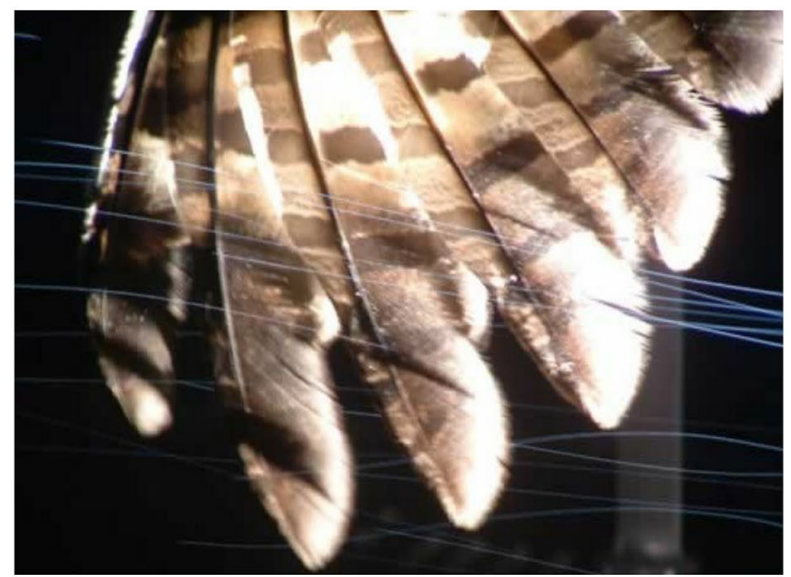

(b)

Fig. 8 a Wind tunnel setup; b smooth streamlines at the great horned owl wingtip [19]

Fig. 9 Flow visualization of the wingtip vortex for the slotted wing of a jackdaw. a Positioning of the measurement planes relative to the bird. b Transverse flow field cutting the tip vortex in gliding flight. c As b, but at mid-downstroke during flapping flight. d On-wing flow field at the slotted wing tip in gliding flight [20]
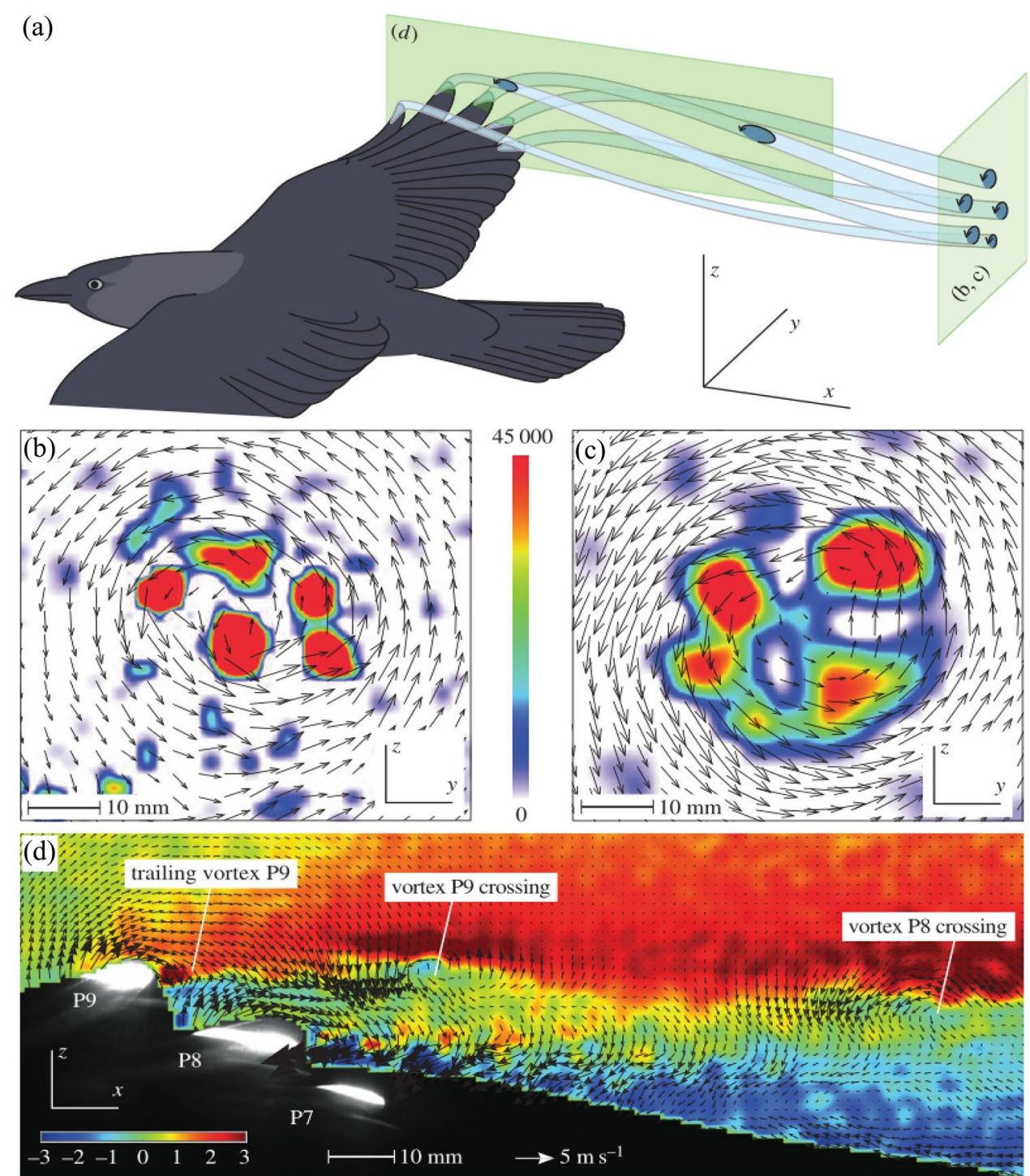
Fig. 10 a The propeller setup for wind tunnel tests; $\mathbf{b}$ polar diagram of lift coefficient $\left(C_{L}\right)$ versus drag coefficient $\left(C_{D}\right)[23]$

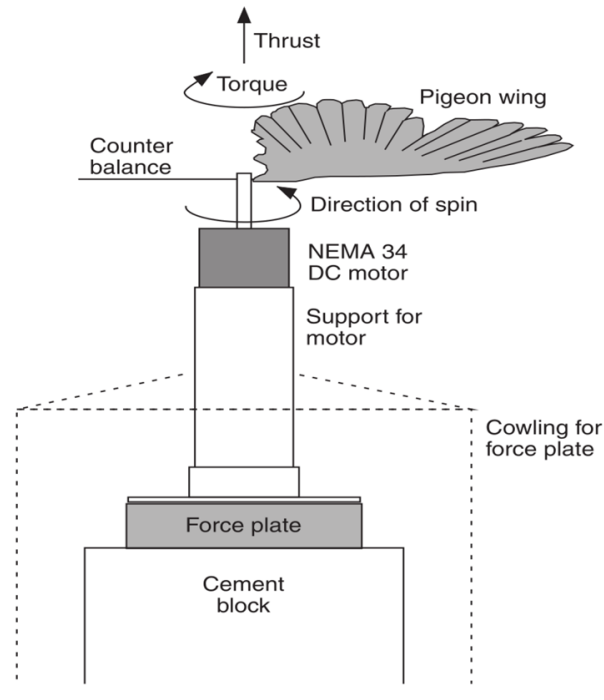

(a)

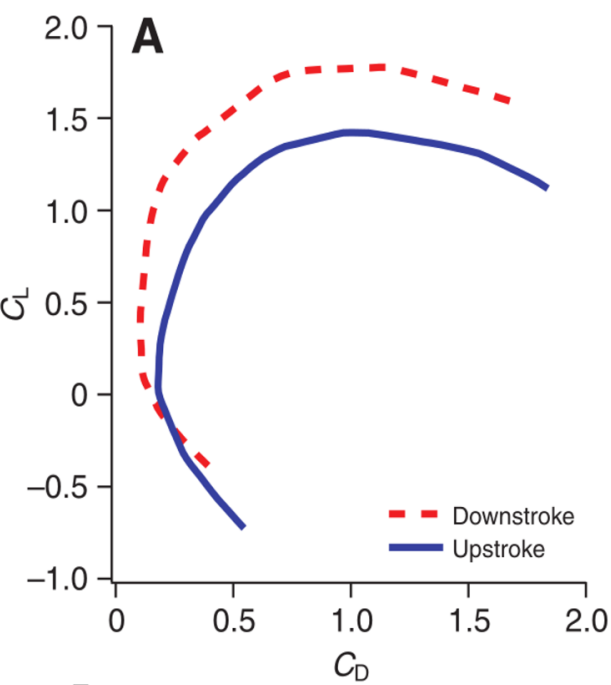

(b)

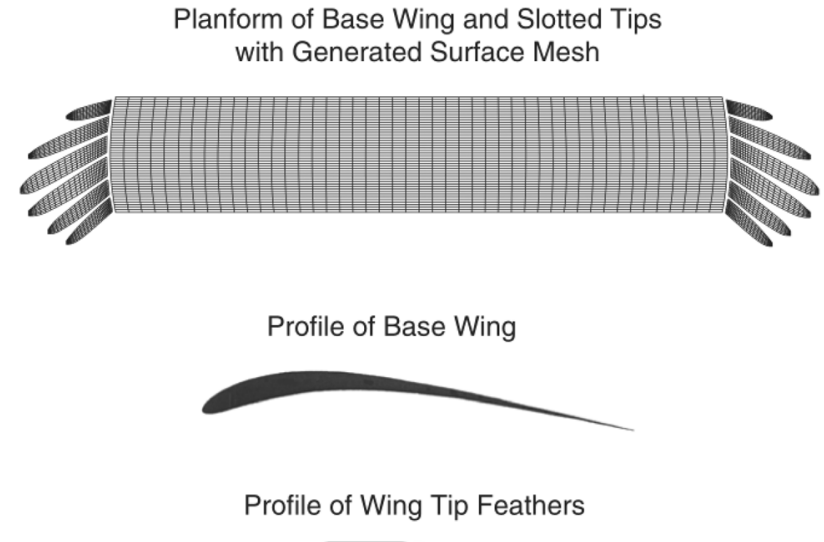

(a)

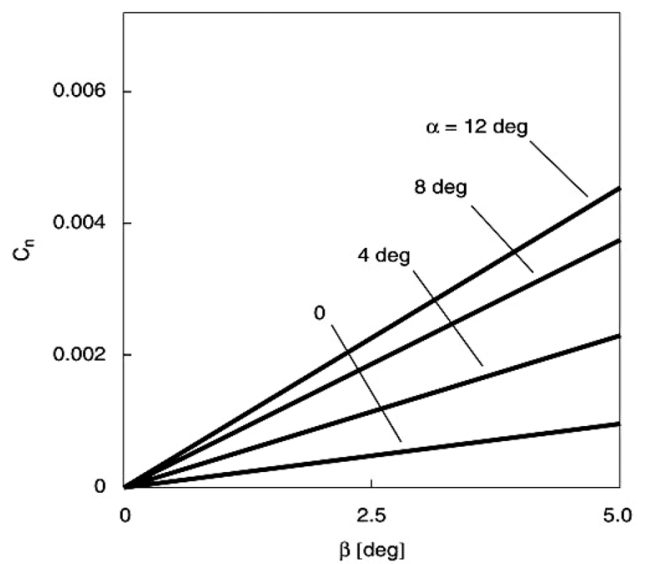

(b)

Fig. 11 a Generic bird wing with slotted tips. The planform and the generated surface mesh as well as the profiles are shown, used in the aerodynamic computation of the yawing moment characteristics; $\mathbf{b}$ yawing moment characteristics of the wing with a sweep in slotted tips [24]

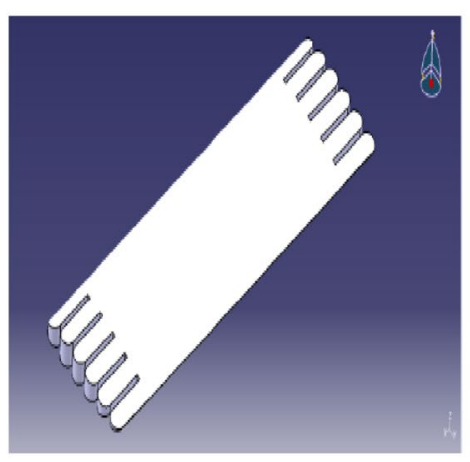

(a)

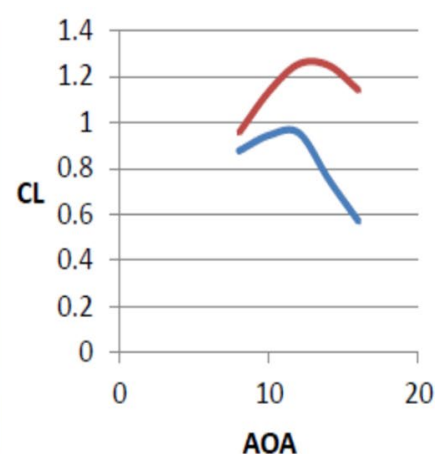

(b)

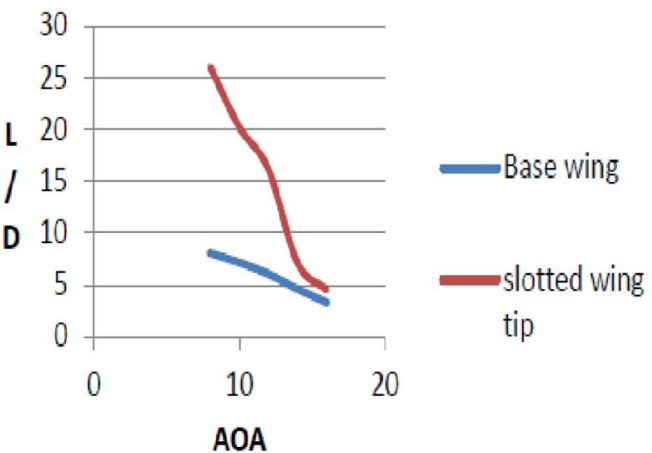

(c)

Fig. 12 a The CAD model: rectangular wing with slotted wingtips; $\mathbf{b}$ lift coefficient and $\mathbf{c}$ lift-to-drag ratio of the base wing and slotted wing versus angle of attack (base wing: blue line, slotted wing: red line) [25] 
Fig. 13 The is bird wing compared with the simplified wing in the bird wing for the lift line model [26]
Fig. 14 Lift-to-drag ratio and drag polar for an elliptic wing versus base wing with five twisted feathers [26]
Fig. 15 Lift-to-drag ratio and drag polar for an elliptic wing versus base wing with different canted feathers [26]
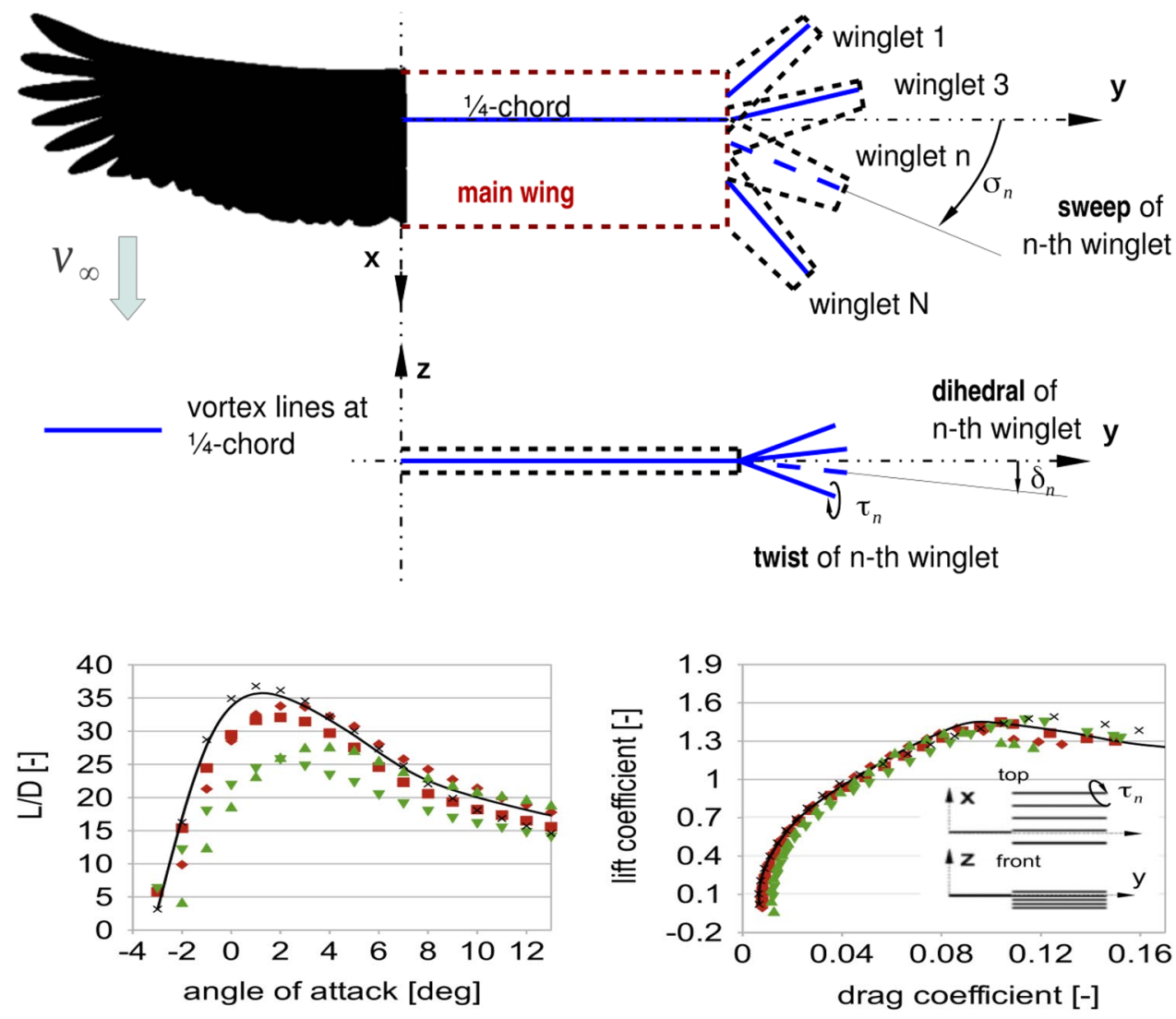

$\times$ no twist

- elliptic wing

$\nabla=-2^{\circ}$

$\triangle \quad \tau=2^{\circ}$

$-\tau=4^{\circ}$
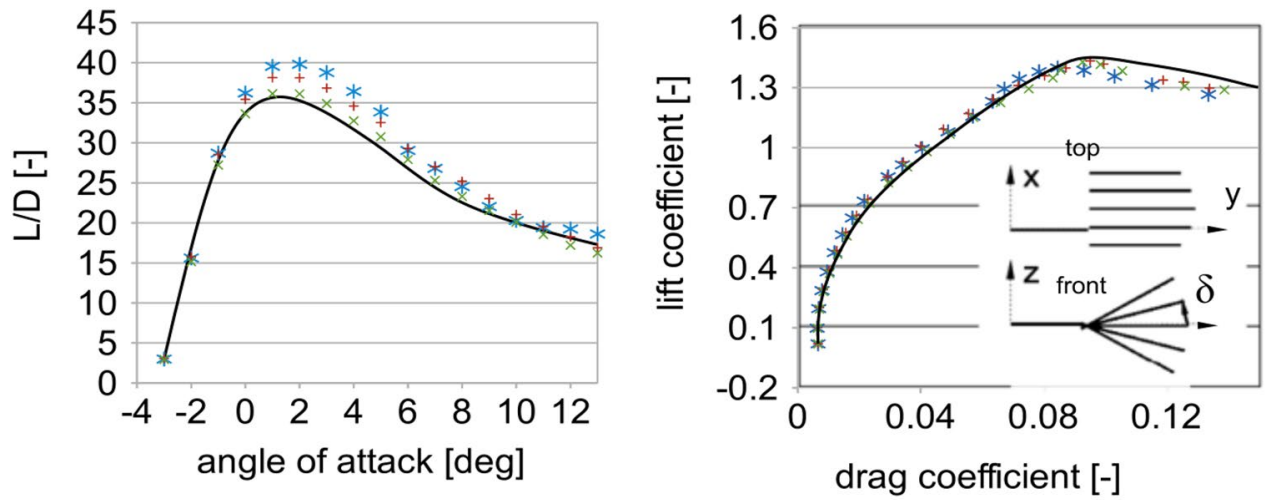

- elliptic wing

$$
\times \delta=0^{\circ}+\delta= \pm[0,15,30]^{\circ} * \delta= \pm[0,30,60]^{\circ}
$$

Mitchell and Jacob [29] investigated the influence of slot length on the aerodynamic properties of wings (Fig. 20). Wings with various tip configurations were compared to a control wing to observe the variations in aerodynamic performance. Figure 20 shows that wingtip slots have a small effect on the lift of a wing. The gains and losses were rather marginal. He presumed tip slots were affected by the operating environment and design purpose and could prove beneficial in the right situations.

Smith et al. [30] examined the aerodynamic characteristics of slotted wings with multiple configurations. When observing lift force, as seen in Fig. 21, they found a remarkable increase of straight slotted wing than base wing with the same area. It was found that slotted wings with appropriate 


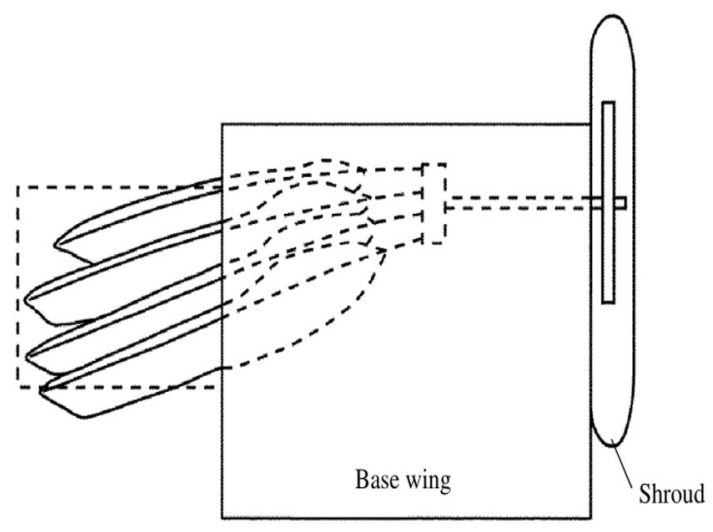

Fig. 16 A top view of the wing balance with the base wing and the feathered tip mounted on it [27]

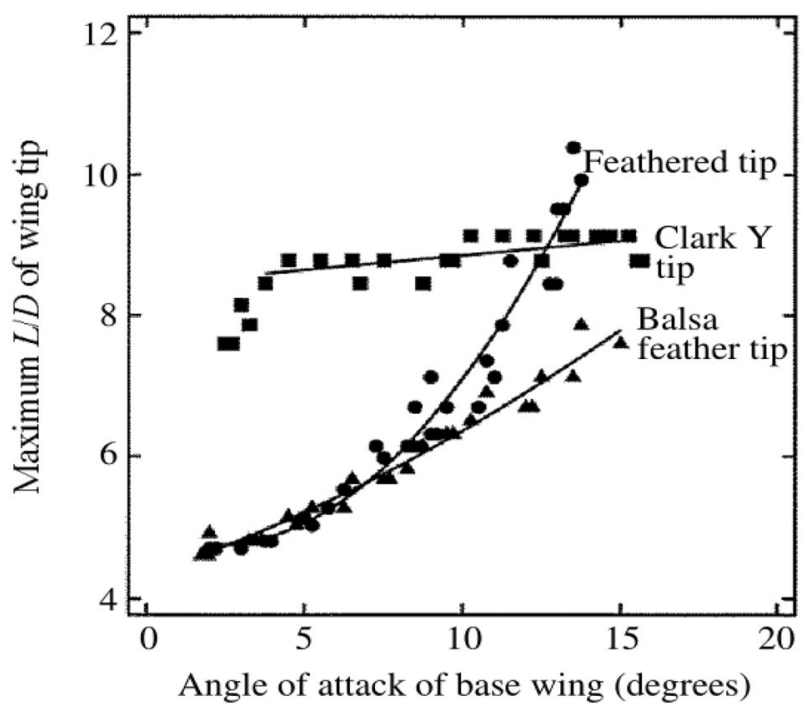

Fig. 17 The effect of the angle of attack of the base wing on the maximum lift-to-drag ratios of three tips [27]

cant angle and twist angle can enhance wing lift and lift-todrag ratio.

Assisting with wind tunnel experiments, our team comparatively studied the unsteady aerodynamic behavior of a flapping feathered wing and a flapping membrane wing [31]. Amazingly, the slotted wingtip formed in the upstroke of the feathered wing can alleviate the negative lift, forming a greater average lift force.

Siddiqui et al. [32] evaluated how cant angles and flexibility impact slotted wings' behavior (Fig. 22). They aimed to figure out the best-performing wingtip configuration among tested wings. The results revealed that rigid wing with curved tip had optimum aerodynamic characteristics, and flexibility has a positive impact on drag reduction and stall delay.

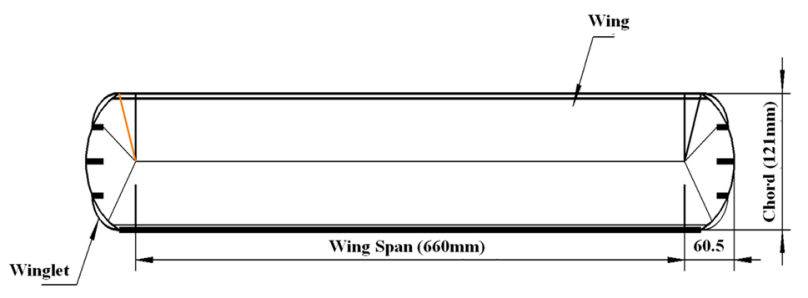

(a) Plan View

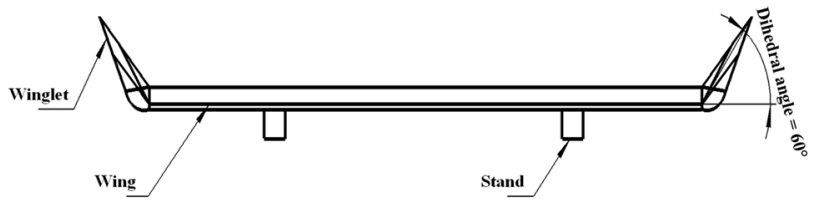

(b) Front View

Fig. 18 Wing model for wind tunnel test: rectangular wing with wingtip adapter to change cant angles [28]

To figure out the influence of cant angle, slot width, and

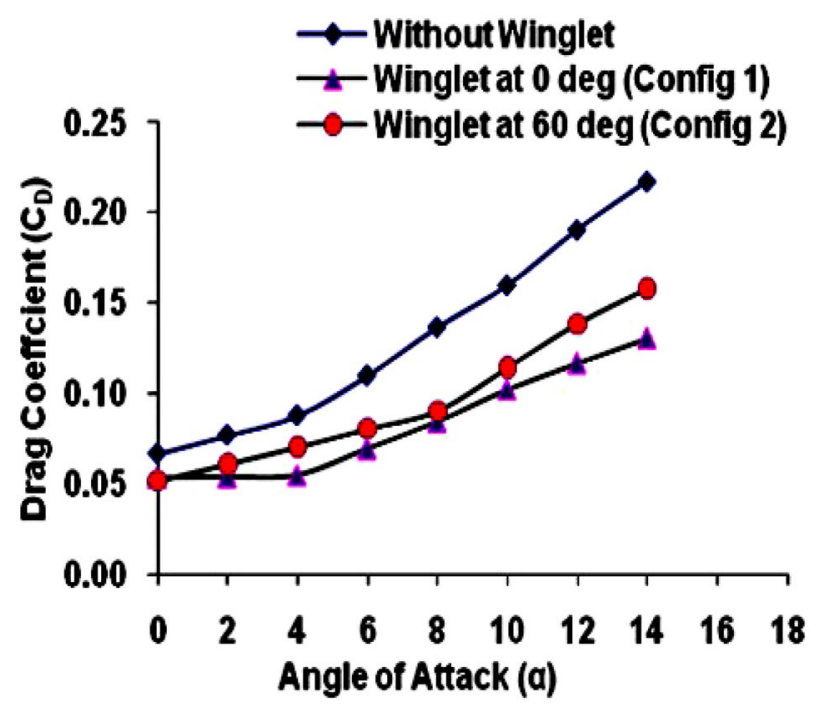

Fig. 19 Drag coefficients versus angle of attack for three aircraft wing models [28]

angle of attack of wingtip on the aerodynamic properties of wings, Lynch et al. [33] conducted wind tunnel experiments on wings with plenty of wingtip arrangements (Fig. 23). As depicted in Fig. 24, for a planar wing with a slot width of $20 \%$, the mean coefficient of lift in the pre-stall region was increased by $7.25 \%$, and the maximum coefficient of the lift was increased by $5.6 \%$ compared to a configuration without tip slots. The outcome also supported the assumption that the effect of slot width is independent of planarity/nonplanarity of the wingtip. Figure 25 presents the calculated 
Fig. 20 a Wing model used in wind tunnel tests; $\mathbf{b}$ wingtips: rounded, feather, and long feather; c measured lift coefficient versus angle of attack at $1.6 \mathrm{~m} / \mathrm{s}$ [29]

Fig. 21 a Wing model with five winglets; effect on $\mathbf{b}$ lift coefficient and $\mathbf{c}$ lift-to-drag ratio versus angle of attack (Exp refers to wind tunnel test, CFD refers to Computational Fluid Dynamics. The numbers in brackets represent different wing configurations: 0 , base wing; 1 , straight slotted wing; 2 , the slotted wing with a cant angle [30]

Fig. 22 Details of slotted tip used in wind tunnel tests [32]

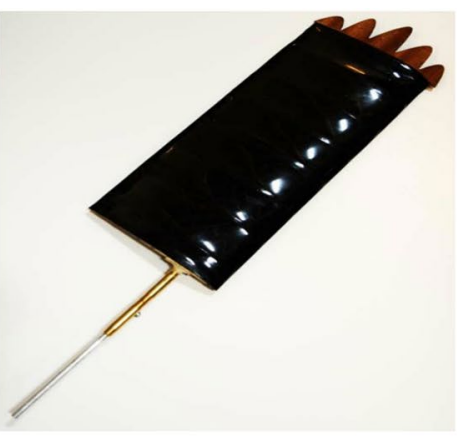

(a)

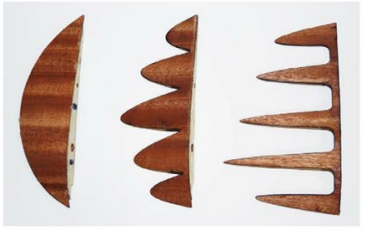

(b)

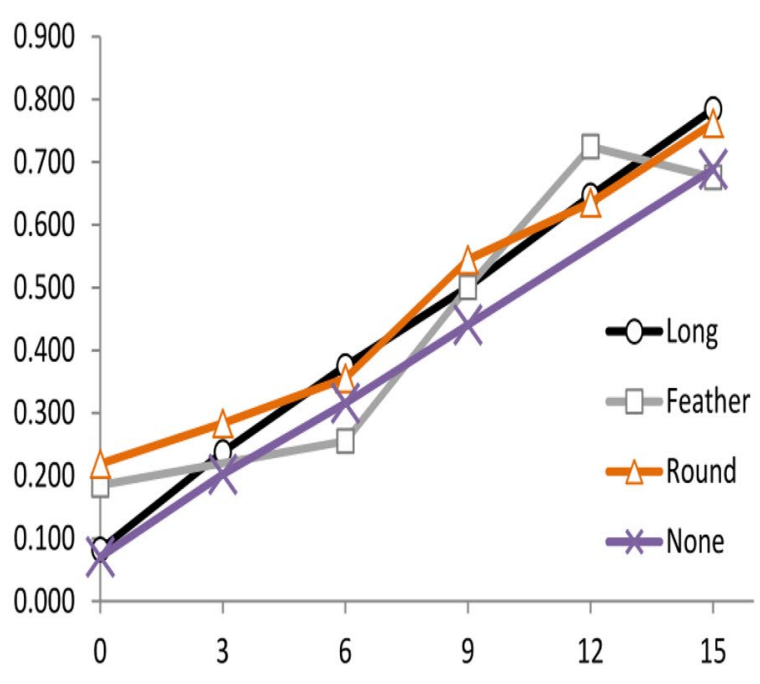

(c)

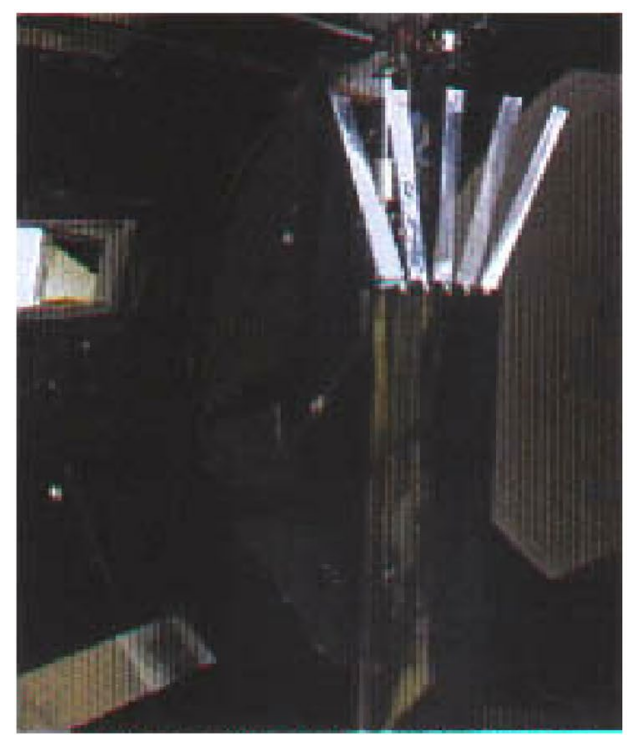

(a)

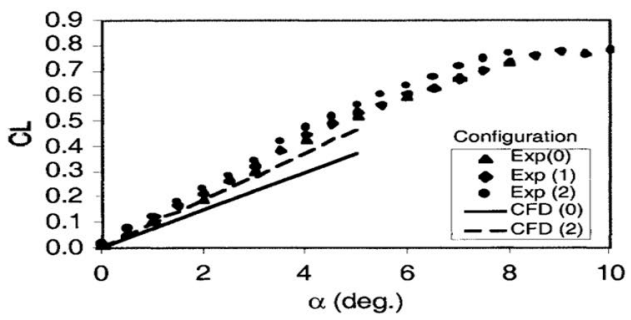

(b)

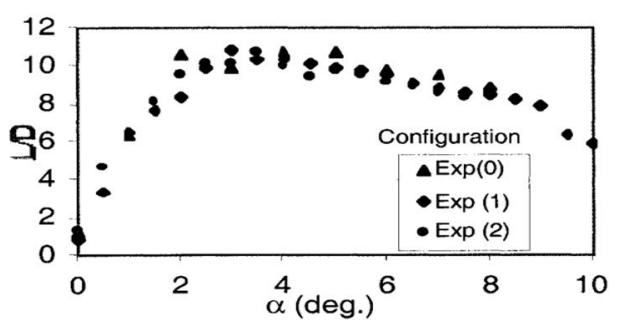

(c)

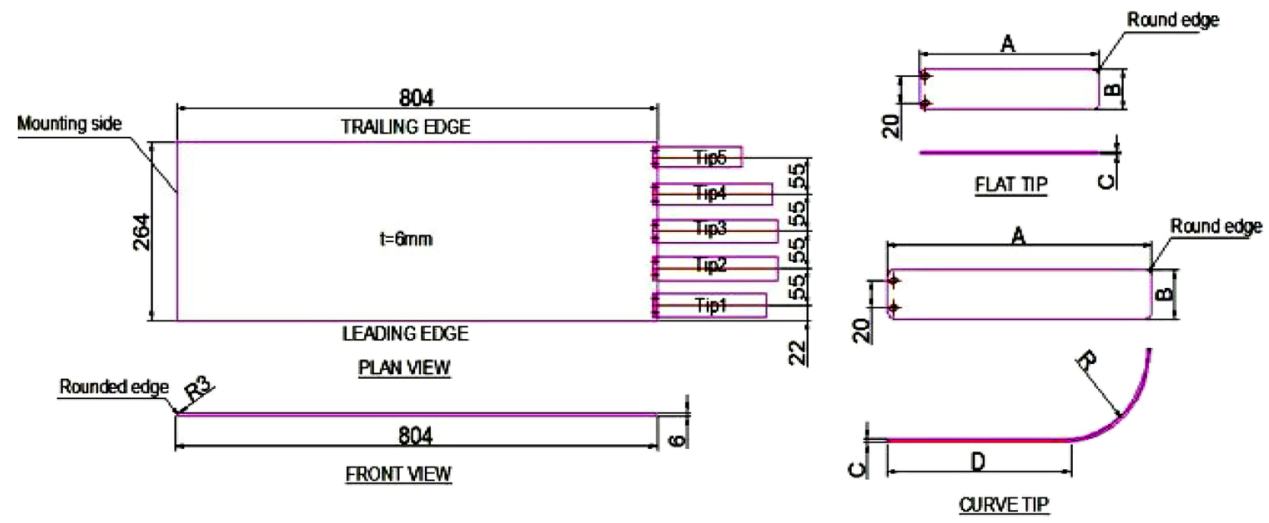


values of $C_{D i}$, dedicating that induced drag is reduced by a significant amount in the pre-stall region, ranging from 17 to $40 \%$, by switching from the planar to the nonplanar configuration.

Hui et al. [34] designed five bionic slotted wing configurations (Fig. 26), aiming to study the impact of the number of slots and slot width on the wingtip vortex characteristics. In Fig. 27, the vorticity level of the wingtip vortex near the wake can be substantially suppressed by improving the slot number.

\subsubsection{Engineering Applications of Wingtip Slots}

In addition to these wing model tests, some researchers have applied slotted bionic wing tip feathers to aircraft. Coiro et al. [35] designed bionic multiple-winglets to improve turning and soaring characteristics of motor-glider as well as hang-glider (Fig. 28). After optimizing the geometric parameters of the slotted wingtip, the performance of the hang glider is improved by about $15 \%$ compared with that of the elliptical wing [36].

Wing-grid [38] is a series of winglets mounted on the wingtip (Fig. 29). Aircraft equipped with wing-grid can quickly dissipate wingtip vortex as well as change lift distribution, thus lessening the induced drag. However, this kind of wing is difficult to adapt to the changing flight conditions, so it has not been widely implemented.

In 2019, Airbus launched a new concept aircraft called "bird of prey" [39], as can be seen in Fig. 30, bionic wingtip slots are distinctly applied. It aims to inspire the next generation of aviation engineers' design inspiration, broaden their ideas, and put forward the potential of Bionics in aircraft design.

Extensive researches on "wingtip sails" are studied for mimicking slotted wingtip where such applications are feasible in practical applications like transport planes as well as ground effect vehicles [40-52]. Similar to the slotted wing, Spillman [40] put forward the concept of "wingtip sails", that is, a plurality of thin pieces installed at the tip of the wing. The wingtip sails with twist and taper can alter airflow direction around the wingtip to generate extra thrust, and also suppress the strength of the wingtip vortex. Furthermore, the wind tunnel tests on a wing equipped with three wingtip sails showed that the wingtip sails could acquire a 30\% reduction for induced drag [41, 42]. Subsequent flight experiments of wingtip sails on Paris MS 760 Trainer Aircraft, Piper Pawnee 235, and Cessna Centurion proved their advantages (Figs. 31 and 32). A wingtip sail is a device that can both mitigate fuel consumption and provide a structural benefit to the wing [43, 44].

Chen and Qi [45, 46] optimized the wingtip sails for a transport aircraft with rectangular wings, and the results

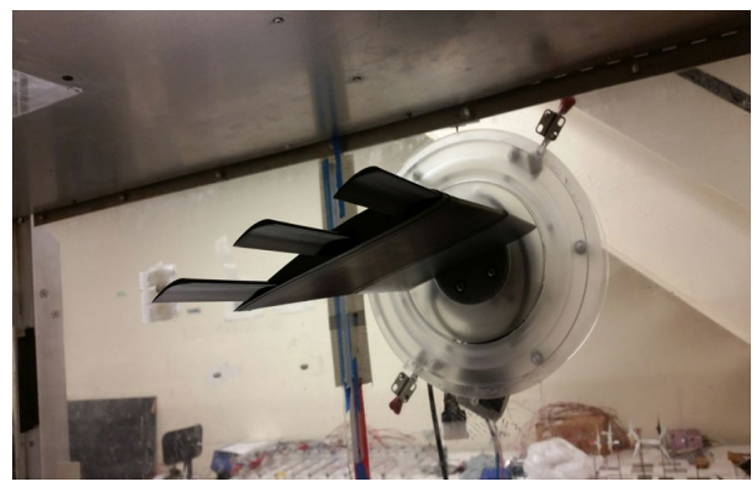

Fig. 23 Experimental setup with slot width $\gamma=40 \%$ and $(0,0,0)$ configuration for angle of attack of wingtip [33]

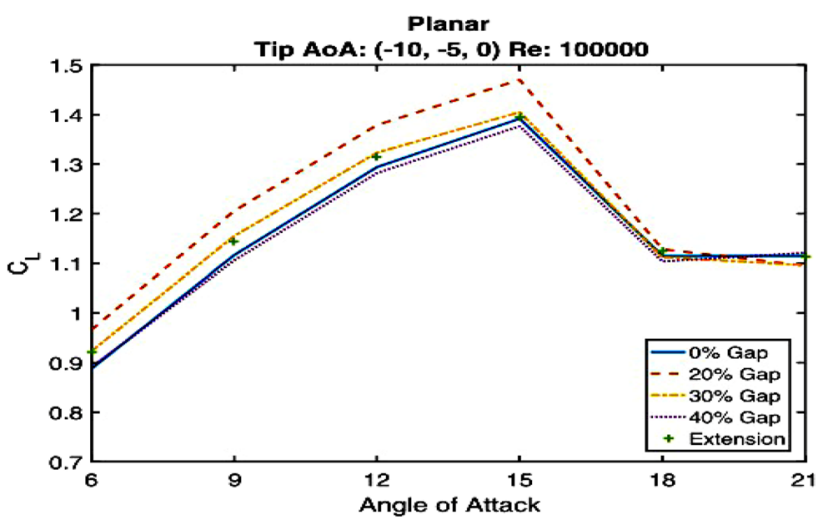

Fig. $24 C_{L}$ versus $C_{D}$ for the planar configuration demon-strating the effect of gap size. $\gamma=20 \%$ produces the greatest lift while incurring small drag penalties for the wing-wingtip system [33]

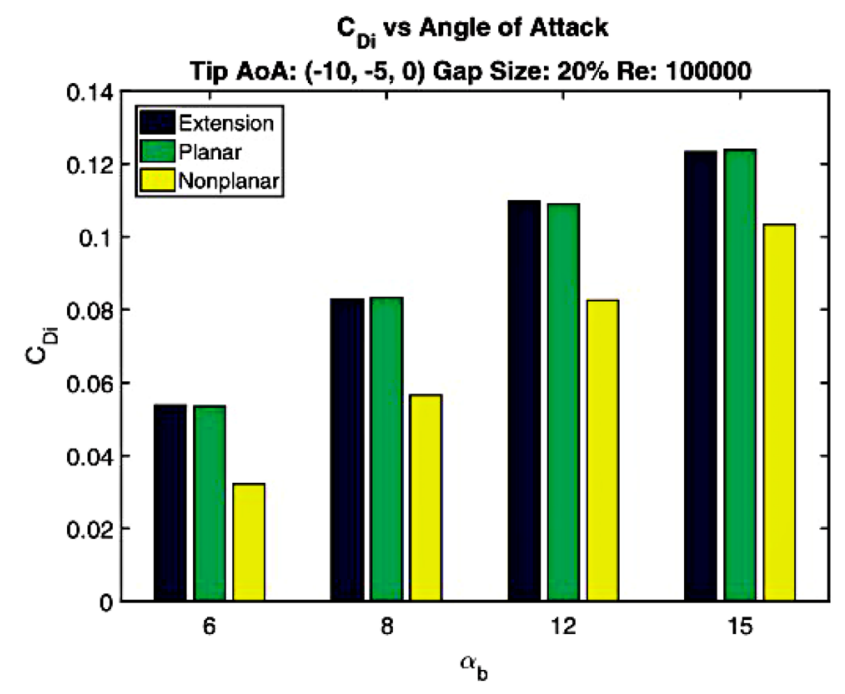

Fig. $25 C_{D i}$ versus $\alpha_{b}$ for planar and nonplanar configurations for pre-stall angles of attack showing a reduction in induced drag when switching to a nonplanar configuration [33] 


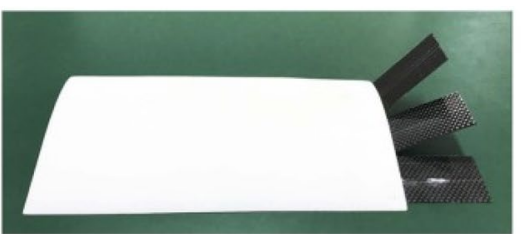

(a) Slotted wing with two slots

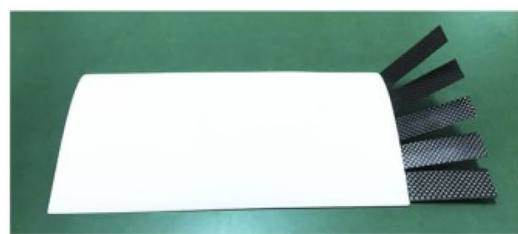

(b) Slotted wing with four slots

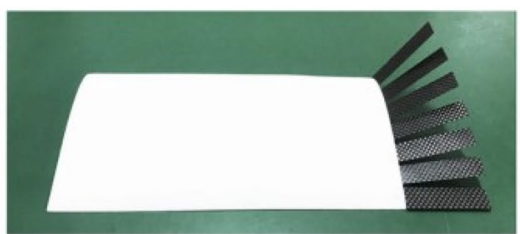

(c) Slotted wing with six slots

Fig. 26 Bionic slotted wings with different slot numbers [34]
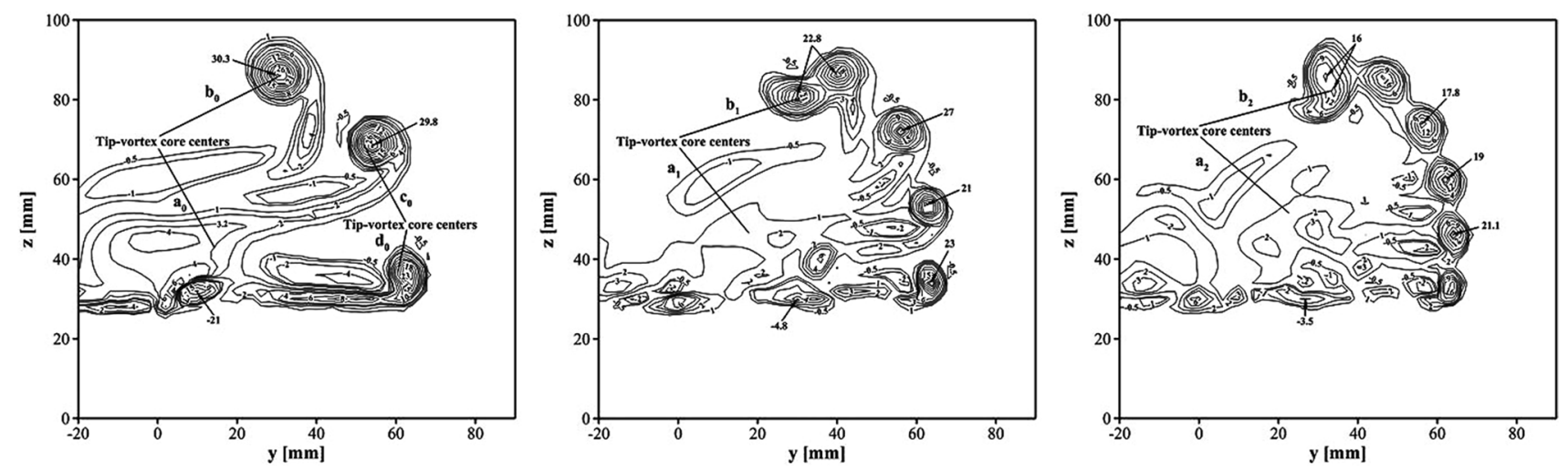

Fig. 27 Dimensionless vorticity contour of bionic slotted wing layouts at $x / c=1.04$ for $\alpha=12^{\circ}$ [34]

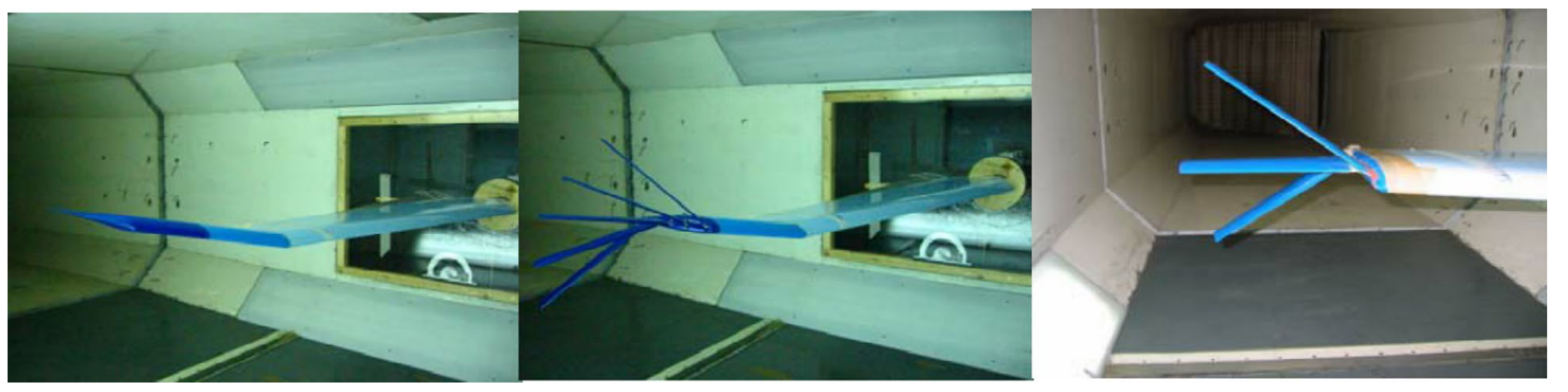

Fig. 28 Some tested Wing configurations: total wing; short wing with five winglets; short wing with three winglets (from left to right) [37]

showed that the tip sails have observable efficiency in reducing induced drag. To study the potential use of adaptive wingtip sails for the reduction of induced drag through variations of cant angles, a total of 55 configurations were chosen with different cant angles by Cerón-Muñoz et al. [47, 48] and tested their performance in a wind tunnel (Figs. 33 and 34). They obtained the best layout of wingtip sails for optimum aerodynamic characteristics in each flight condition.

Al-Atabi et al. [49, 50] achieved a reduction of induced drag about $35 \%$ after installing wingtip sails on a
NACA0012 wing (Fig. 35). While the induced drag could be further reduced by a larger incidence angle or more wingtip sails. Unfortunately, this also affected the longitudinal static stability of the wing, which tended to be mitigated by moving sails near the trailing edge of the wing.

Large aquatic birds, like pelicans, can glide over the water with the help of separate primary feathers to catch fish. Inspired by this, Sun et al. [51, 52] experimentally studied the aerodynamic properties of ground-effect aircraft with wingtip sails (Fig. 36). As the wing approaches the ground gradually, both trapezoidal sails and elliptical sails show the contribution to lift increase and drag reduction. 


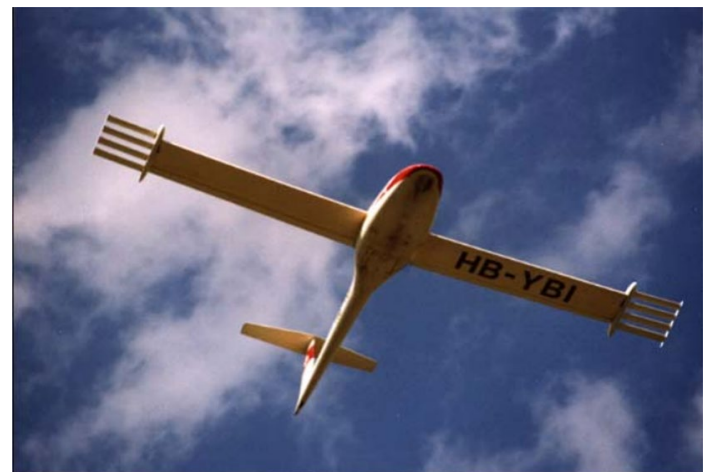

Fig. 29 Flight test of wing-grid concept [38]

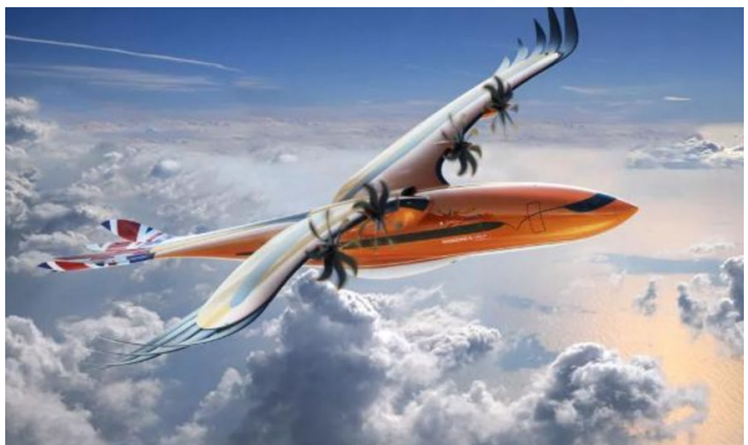

Fig. 30 Concept aircraft called "bird of prey", announced by Airbus [39]

Moreover, there are many UAVs' (Unmanned Aerial Vehicle) design work were inspired by wingtip slot [53-56]. Inspired by the bionic slotted wingtip concept, Shelton et al. [56] demonstrated that active multiple winglets can be a substitute for ailerons. With this kind of slotted wing fitted to the UAV "Dragon Eye", its range and endurance can be enhanced by $40 \%$. Moreover, it can present gust alleviation for improved handling qualities and sensor performance (Fig. 37).

The "BionicSwift" [57], designed by the Festo company in Germany, adopts simulated feather wings which can be overlapped and unfolded. During the upstroke, the wings' feathers split to form wingtip slots (Fig. 38), allowing air to flow through the wings to produce greater lift; The slots are closed during the downstroke, generating more flight power.

\subsection{Basic Theories of Induced Drag and Wingtip Devices}

The lift generated by the wing inevitably produces vortex sheets in the wake near the wingtip, which will cause the streamline to curl to form the wingtip vortex and generate a downward velocity component (i.e. downwash). This

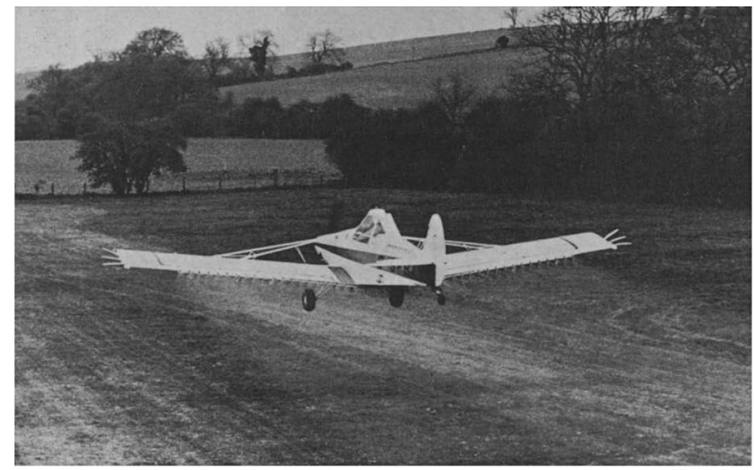

Fig. 31 Piper Pawnee aircraft equipped with wingtip sails [41]

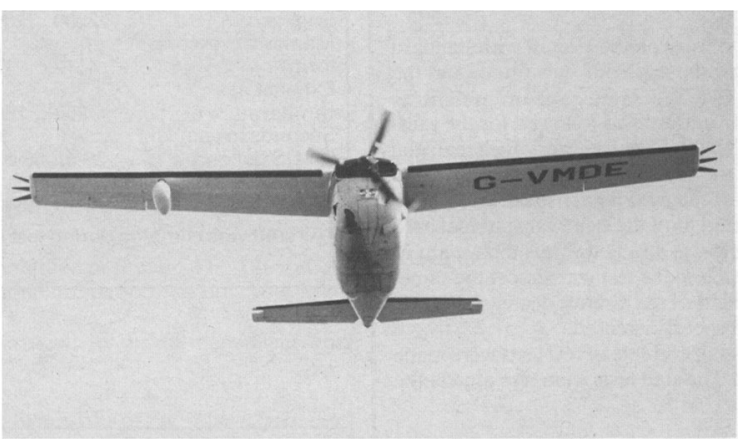

Fig. 32 Cessna Centurion with wingtip sails [43]

downwash reduces the effective angle of attack of the wing and inclines the lift vector backward, producing an additional force component (i.e. induced drag) in the drag direction $[58,59]$. The reduction of induced drag is essential for aircraft. For instance, the induced drag generated by a transport aircraft in the cruise phase accounts for $40 \%$ of the total drag, and even $80-90 \%$ of the total drag during takeoff [60].

The direct way to minimize the induced drag is to enlarge the wing span, while this will bring extra parasitic drag and structural weight. Another feasible method is to use the wingtip device, designed for weakening the strength of the wingtip vortex and thus reducing the induced drag [61]. For birds flying with slotted wingtips, the lift can be increased and stalling delayed in several ways, the most important of which is the wingtip slot [62]. Consequently, how to design the wing by imitating the structural characteristics of bird wingtips has become a major challenge for flow control of aircrafts' wingtips.

Owing to the superior advantages of the birds' wing in suppressing wingtip vortices, in addition to multiple winglets, wing-grid and wingtip sails, many other bird-like wing structures for minimizing induced drag have been explored (Fig. 39). Whitcomb's achievement [63], the (A) Whitcomb winglet, marks the first time the wingtip device was formally 


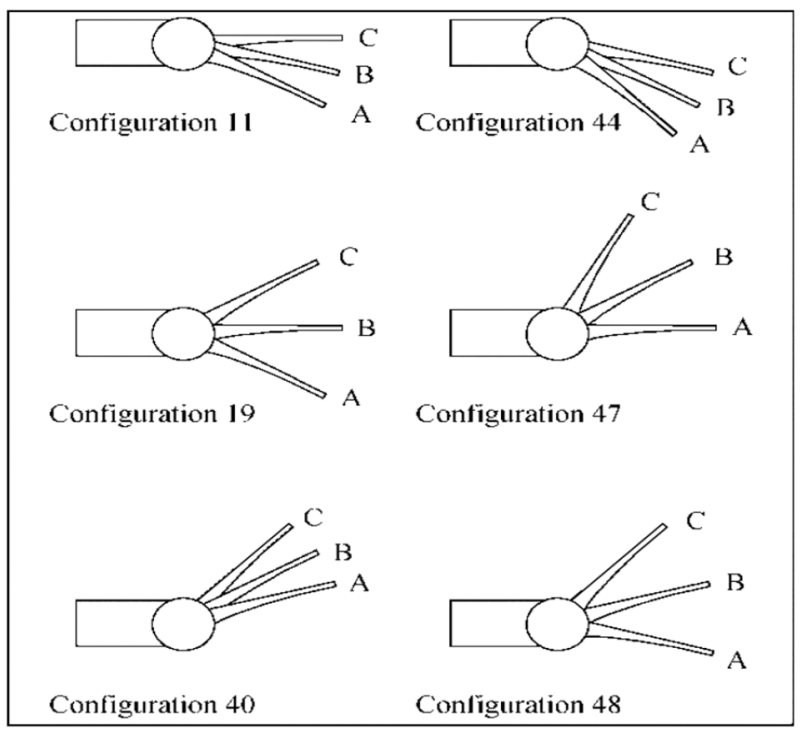

Fig. 33 Optimum wingtip sails layouts, where $\mathbf{A}$ is the leading sail, $\mathbf{B}$ is the central sail, and $\mathbf{C}$ is the trailing sail [47]

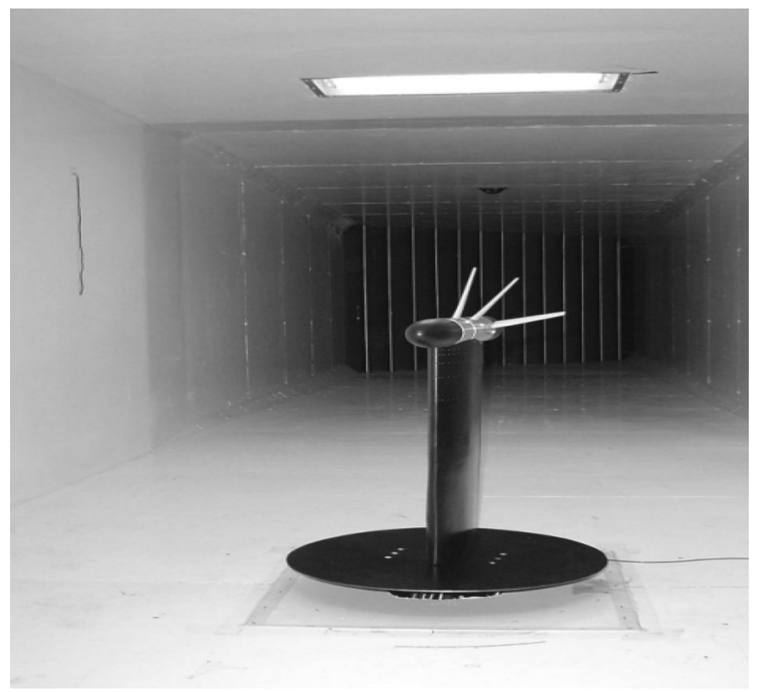

Fig. 34 Wing model equipped with wingtip sails placed in the wind tunnel [48]

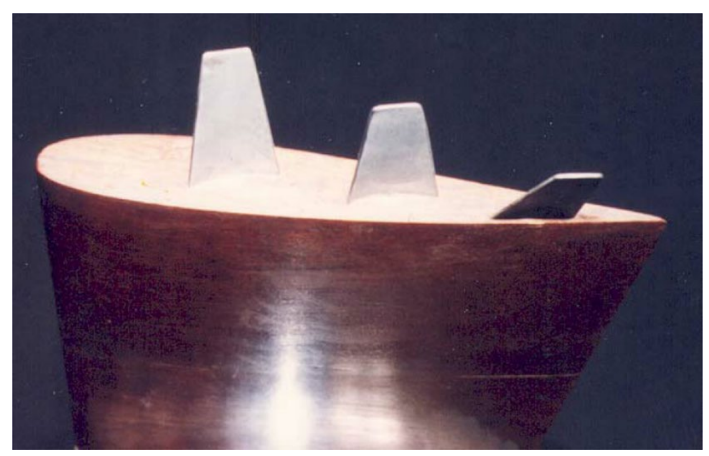

Fig. 35 NACA0012 wing mounted with three wingtip sails [49]

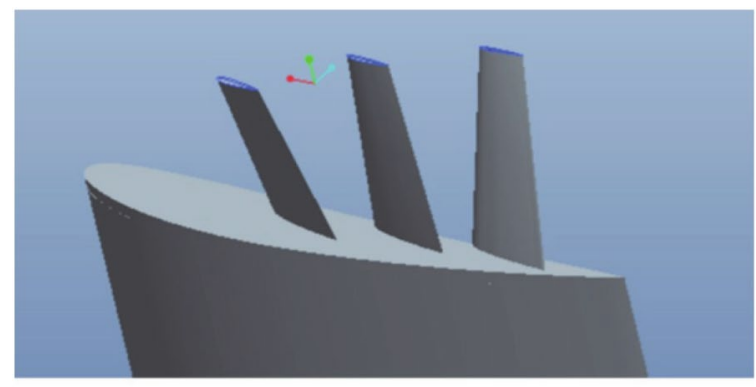

(a) Long tip-sails

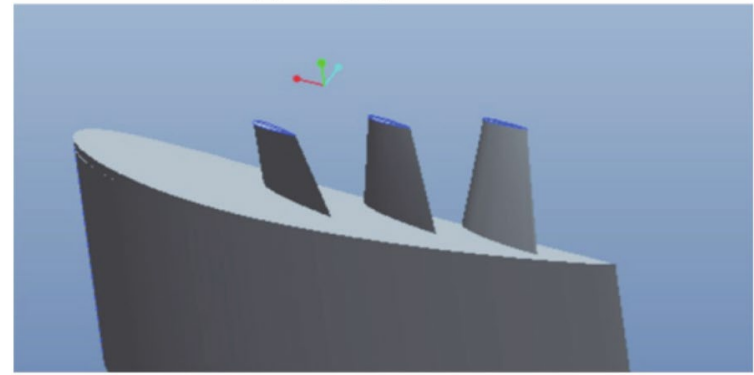

(b) Short tip-sails

Fig. 36 Wings equipped with long tip sails and short tip sails [52]
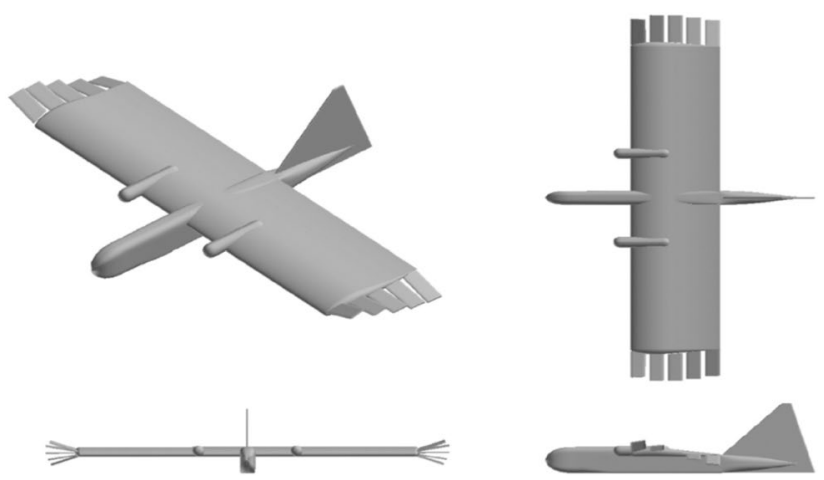

Fig. 37 Three views of "U.S. Marine Corps Dragon Eye UAV" configuration with bionic multiple winglets [56]

applied to large and heavy aircraft. He combined advanced airfoil concepts into a swept, tapered planform that would interact with the wingtip airflow to reduce drag. Some variant designs of winglets have appeared over the next years. The (B) tip fence is an endplate at the wingtip to reduce the occurrence of tip vortex and induced drag $[64,65]$. It is easy to install on the wing without any modification to the shape of the wing itself. However, it increases the aspect ratio of the wing, and it no longer plays an active role with the increase of angle of attack. The tip fence must be so large that the augment in skin friction drag due to an excessive wetted area far outweighs the drop in induced drag. Likewise, additional area caused by the (C) canted winglets enhances parasite drag and may cut aerodynamic performance at high angles of attacks in addition to the increased 


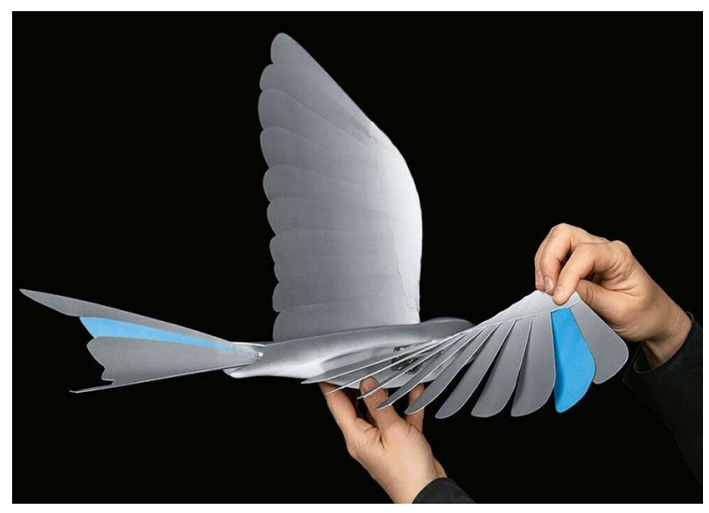

Fig. 38 The bird-like robot "BionicSwift", designed by Festo [57]

weight to the device itself [66-68]. The (D) vortex diffuser and the $(\mathrm{H})$ tip turbine aim to diffuse wingtip vortices. The idea behind them is that, by expanding the core radius of the vortex, the contained kinetic energy, and thus the induced drag in high-lift/high-angle-of-attack configurations, is reduced. Unfortunately, it is not possible to achieve the exact symmetry of the vortex roll-up for such devices $[69,70]$. The additional purpose of vortex diffusers is heat transfer enhancement, which increases with the angle of attack and decreases with the diffuser angle and with Reynolds number [71].

The (E) raked tip improves aerodynamic performance by increasing the wing's effective aspect ratio. It yields the best lift-to-drag ratio at low angles of attacks $[72,73]$. The (F) blended winglet is defined as a wing-like surface encompassing a blended surface for enhancing lift and minimizing induced drag [74]. As introduced before, (G) tip sails consist of small sails attached to the wingtip and adjusted according to the flow direction at that region, creating a thrust force and breaking up the vortex system as well as reducing its intensity. The conclusion is that once a particular flight condition has been chosen, the geometry of the tip sail must present a twist and taper ratio (which in turn will cause flow separation). The airfoil must be highly curved at the root and symmetric at the tip [47, 48]. The (I) wing-grid operates as a split wing. It creates small vortices that dissipate the energy of the main vortices and modifies the circulation distribution by wing-wing interference of parallel winglets, while the wing-grid does not change the stall characteristics as well as the bending moment. In some cases, it unexpectedly increases the total drag, such as in wings with large aspect ratios or in low-lift conditions, in which the induced drag is far less important than pressure and viscous drag [38]. Mahmood [75] proved the (J) spiroid winglet are likely to be more effective in drag reduction, which looks like an extended blended wingtip that bends upward by $360^{\circ}$ to form a ribbon. The most fuel-efficient aircraft winglet so for is the closed spiroid winglet. Besides, the application of spiroid winglets can obtain wing stall delay and better post-stall behavior. The research suggests that the spiroid winglet had the greatest lift-to-drag ratio at level flight while having the smallest ratio at other angles of attack [76]. Increased weight and static loads due to spiroid winglet demand aircraft to support higher bending moments and meet flutter and fatigue demands. The (K) delta tip is cited as a simple device to promote aerodynamic characteristic and avoid complications related to winglet implementation. Except for reduction in drag, it has a negligible effect on lift improvement. There are also some novel devices trying to influence vortices around wings, such as split winglets [77], chordwise slots [78], and hollow wingtips [79].

Compared to the above passive control methods on the account of load correction, there are active control strategies that affect the vortexes through the force of actuators. These methods include morphed wings [54, 55], wing blowing (chordwise or spanwise) [80,81], synthetic jets or plasma jets actuators, and are also used in others fields such as wind turbines [58]. The selection of the passive control devices or active control devices depends on the specific situation and the airplane type.

Wingtip devices do not all look the same (as illustrated in Fig. 39); nevertheless, their ultimate goals are always induced drag minimization. It can be concluded that a wingtip device is aerodynamically effective only when the reduction of induced drag exceeds the increase of parasitic drag. Especially, in terms of the research focus of this paper, bionic-slotted wingtips, it is the most imitation of a bird wing.

As previously described, the slotted wingtip is similar in structure to wing-grid and wingtip sails, while it is the integration and improvement of these two wingtip concepts. Slotted wingtips with tip slots spread horizontally and vertically to form nonplanar wingtips. They connect smoothly to the base wing to avoid creating extra parasitic drag. Wingtip slots have considerable application potential in MAVs (micro-air vehicles). MAVs have superiorities of small size, lightness, and concealment [82]. It is similar to the operating environment of birds with slotted primary feathers: close to the ground, high turbulence intensity, and sometimes need to avoid obstacles. Unlike conventional wings, there is no obvious stall point in the lift versus angle of attack diagram of bird wings. In addition, the induced drag of bird wings is greatly lower than the theoretical-induced drag predicted by the aspect ratio [83, 84].

However, as wingtip slots are considered for engineering applications, the construction of a slotted wing layout is quite complex. Due to their intricate aerodynamic interaction, each single winglet must be cautiously adjusted to achieve the required drag reduction effect [85-87]. Therefore, it is necessary to comprehensively and systematically 

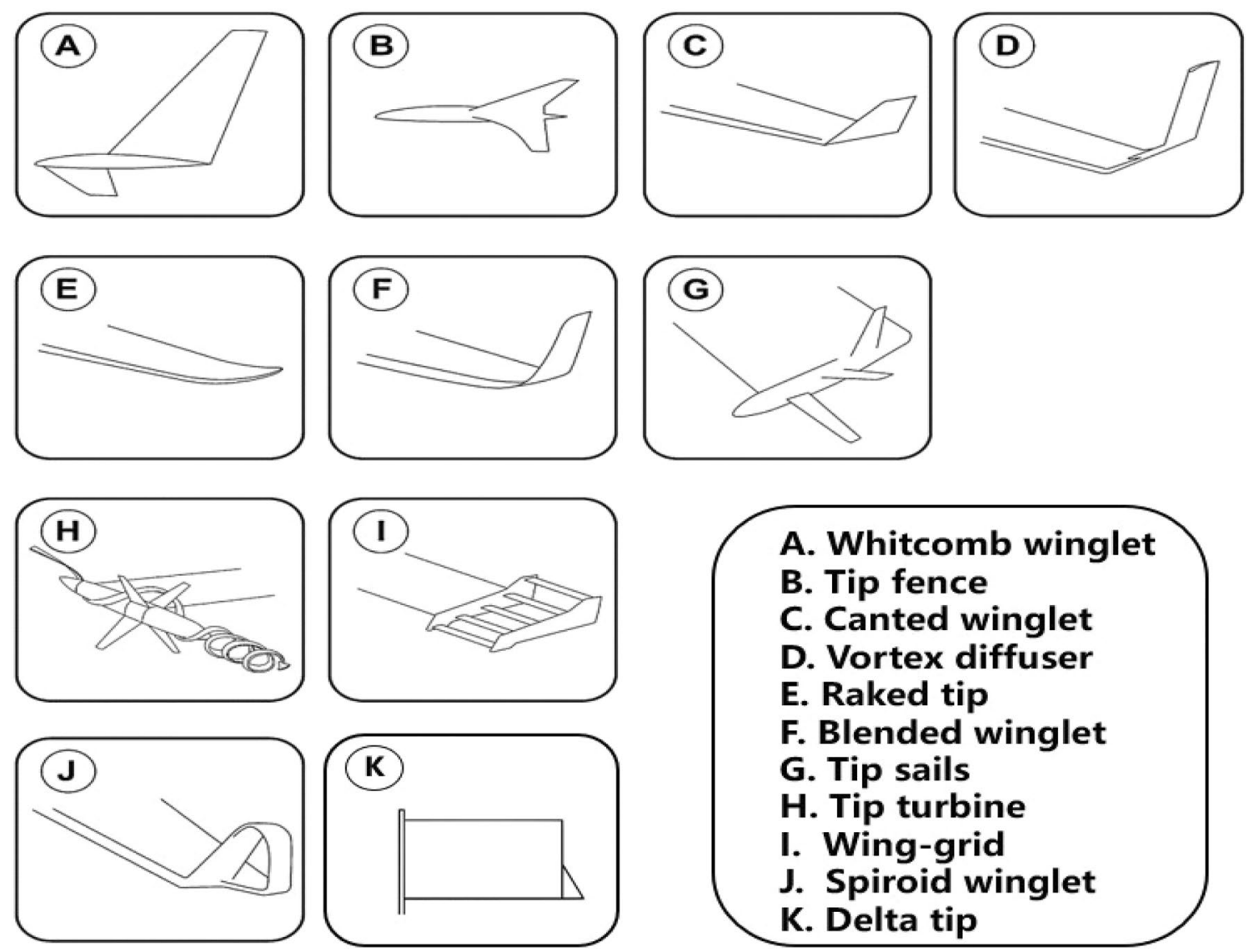

Fig. 39 Different types of winglets and wingtip devices commonly used (adapted from [66])

study the influence mechanism of slotted wingtips' parameters on the aerodynamic performance of wings, which is the main intention of this paper.

\section{Summary of Research Deficiencies}

Scholars have speculated for years about the role wingtip slots played in bird flight. They have proved wingtip slots can break up wingtip vortex, reduce induced drag and improve controlling stability. However, there are still some shortcomings in the consistency of conclusions and the integrity of the research scope. Through the literature review, it can be found that most early explorations of wingtip slots were simply to compare the aerodynamic characteristics between the two groups: wings with or without slotted tips. There are many parameters that constitute the wingtip slots. The specific improvement effect of each parameter on the overall aerodynamic properties of wings and its functional mechanisms are not clear. Particularly, divergent conclusions exist on the lift improvement effect: (a) Some reckon that reasonable arrangement of tip slots on the wing by properly arranging geometric parameters can diminish drag, increase lift and lift-to-drag ratio be enhanced correspondingly $[17,28]$.

(b) other scholars think that even if wingtip slots can reduce the lift, the lift-to-drag ratio can be raised due to a greater reduction of drag [25].

(c) the other studies believe wingtip slots cannot increase the lift and lift-to-drag ratio at the same time, that is to say, wingtip slots can increase lift while drag will be raised unexpectedly [26].

The main reasons lie in one-sided conclusions caused by the incompleteness of research contents and feeble conclusion accuracy due to the limitations of the research methods. The specific analysis and summary are as follows:

(1) Vacancies in current research, especially considering the influencing factors such as unsteady flapping and selfflexibility which are more in line with the real flight status of birds.

The basic parameters of the slotted wingtip are given in Table 2. Specifically, it contains eight structure parameters, 
four motion parameters, and two deformation (flexibility control) parameters.

At present, the coupling impact between wingtip slots and unsteady flapping of bird wings is rarely considered. The reason may be that plenty of scholars believe that wingtip slots structure is more common and prominent in huge soaring birds' wings, and most of the flight of this kind of bird is in the gliding stage, so wingtip feathers mainly play their role in steady state. However, actually unsteady flapping is a normal behavior of the bird, especially in the maneuvering process of birds, such as acceleration, take-off, landing, or sharp turn $[2,88]$. Obvious wingtip slots structure appears on the wings, and birds do not put such structures away. Birds can constantly change wing shape during flight, especially raptors such as eagles and harriers. The distal part of the wing with slotted primaries produces greater thrust than other parts. They need to take advantage of air to gain efficient aerodynamic characteristics of wingtip slots, particularly when they complete key behaviors such as hunting [89].

Our previous research has found that the unsteady effect of wingtip slots with flapping motion played an important role in improving the overall aerodynamic performance [90]. To elucidate the collective mechanism of the flow generated by slotted tips and the lift contribution of each tip, we recovered a time sequence of the slotted wingtip in a whole flapping period from videos of a level-flying bald eagle. The interaction of vortices in time and space leads to wing-wing interference and the flow around slotted tips becomes complicated and unstable. The slotted wing configuration can efficiently convert more energy into the lift. As the flapping frequency increases, the collective feature of the slotted wing with constantly changing gaps can be more advantageous to enhance lift-generation performance (Fig. 40). Moreover, whether the unsteady effect of wingtip slots with flapping has any effect on the improvement of wings' aerodynamic performance and spreading of wingtip vortex remains unclear, so it is worth further exploring.

Based on biological observations, wings' flapping motion and flexibility are two key attributes of low Reynolds number flight [91-93]. In birds, wingtip feathers are flexible. They can twist their wings during maneuvering to obtain optimum thrust or lift [94]. The flexibility of wings is of great significance to reveal the new mechanism of bird flight, it was rarely be discussed in previous work, though. During the flight of birds, the deformation of wings can minimize the power consumption and improve the thrust at the same time $[11,95]$. The slotted wingtips are cantilever structures composed of single primary feathers, which will have obvious deformation during the flight of birds, especially during maneuvering and facing gust. These deformations can dramatically change the aerodynamic shape of wings, and even dynamic deformations can help birds delay or resist the disturbance of gusty wind. Therefore, it is necessary to speculate the flexibility of the wing when exploring the aerodynamic mechanism of the slotted wingtip.

(2) Limitation of temporal research methods. Early wind tunnel tests were weak in the flow field display, while numerical simulations lacked the support of high-precision calculation methods.

(i) The relevant research on wingtip slots was mainly carried out by wind tunnel test and numerical simulation, the Reynolds number ranging from 10,000 to 30,000 . Wind tunnel test was widely adopted among them, due to the imperfect test conditions and flow field display ability at that time, the outcomes were disturbed. Mitchell et al. [29] explained why the lift performance of non-slotted wing obtained by circulation measurement was higher than tip-slotted wing, they proposed that even though wings with tip-slots produced higher circulation values, the strong vortex produced by the wing without tip slots could be more easily measured than the other more dispersed vortices generated by the slotted wing.

(ii) A similar situation was encountered in numerical simulation. It was limited by the solution method of CFD and the development of computer techniques. Smith [30] and Sachs [24] applied the open-source programs in solving flow field, namely Cobalt60 unstructured code and FLM-Eu Code, whose internal core algorithms were based on finite volume method solving Euler equations; however, the viscosity of the fluid is ignored in the solution of Euler equation, and there seem to have a big deviation in obtained data [96].

Nowadays, the Navier-Stokes equation (abbreviated as NS equation) has been widely adopted in computational fluid dynamics, which will take viscosity into account and more in line with the authenticity of fluid flow contrast to the Euler equation [9799]. Furthermore, although Saiteja and Suresh [25] applied the NS equation in numerical simulation, the flow field was solved in a laminar flow state, ignoring the influence of turbulence. As a matter of fact, turbulence is a kind of wave phenomenon appearing in the velocity fluctuation area, its existence impels the fluid medium to appear several phenomena, like slowly exchanging momentum and energy with each other, changing concentration, and so forth [100102]. Therefore, in numerical simulation, the influence of turbulence should be taken into consideration with regard to choosing the solution model.

(iii) Moreover, the drag characteristic of the wing is a crucial index to measure its aerodynamic behavior, the majority of experiments measured the drag produced 
by the whole wing. However, the slotted tip is only a part of the wing, and its effect is mainly on the wing near the tip.

Obtaining accurate drag force is a very tough task with the aid of wind tunnel experiments or numerical simulations [103, 104]. Traditionally, computational fluid dynamics (CFD) computation utilizes surface integration, or the near-field method to predict drag, the pressure and stress tensor on the surface of the aircraft body is integrated for the drag prediction $[105,106]$. However, it has been pointed out that the total drag calculated by the near-field method contains spurious drag resulting from numerical diffusion, which cannot be separated from the total drag [107-109]. Since tip slots play a major part in the variation of induced drag, it is necessary to decompose experimental drag to study the variation of induced drag components under assorted tip configurations using a more accurate drag calculation method.

(iv) For analysis of aerodynamic mechanism, few studies focused on the formation and development of vortices. Earlier researches put emphasis on data comparison of aerodynamic force and moment obtained from wind tunnel test and numerical simulation, in the same experimental conditions, differences of aerodynamic forces can be analyzed among several groups of wings with varying wingtip slots parameters. Whereas, as introduced in Sect. 2.2, vortex analysis is also crucial for exploring the aerodynamic mechanism, even contributes to discovering deeper mechanisms in contrast to aerodynamic force comparison [110].

\section{Strategies and Discussion on Research Prospect}

Aerodynamics of bird airfoils and wings can be classified into three categories. First, the analysis of airfoils/wings of birds as a fixed rigid body; second, birds airfoils/wings in flapping phase; and third, flexible airfoils/wings in nonflapping flight [15]. Combined with the conclusions and deficiencies of the literature investigation, it is reckoned that subsequent research could extend to geometric and kinematic parameters of wingtip slots structure in unsteady state, and flexibility should be given priority as well.

Based on the previous achievements in steady state, further explorations and expansion of the influence factors are still needed, more attention should be poured into the application of the more accurate techniques to acquire drag of slotted wings. The flow field integration method, which can extract the drag component and evaluate the variation of induced drag separately, is highly recommended. As for the aerodynamic mechanism of wingtip slots, the emphasis can be placed on the study of the formation, development, and evolution of wingtip vortices on slotted wings varying from geometric structures and spatial distribution of winglets. The application of PIV (Particle Image Velocimetry) technology in wind tunnel tests and post-processing of numerical simulation can be adopted to realize flow field visualization and vortex analysis. Based on the research background of wingtip slots and the aerodynamic characteristics concerned, the research roadmap is proposed for discussion, as depicted in Fig. 41. The research prospects are considered to be divided into three parts: (1) Research on geometric parameters of wingtip slots in steady state; (2) Research on geometric parameters and kinematic parameters in unsteady state; (3) Research on aeroelasticity of wings considering the flexibility of slotted wingtips. Finally, as illustrated in the first column of Fig. 41, the authenticity and integrity of research can be guaranteed by combining the steady, unsteady, and flexible achievements in sequence.

\subsection{Geometric Parameters in Steady State}

In steady case, geometric parameters of wingtip structure: the shape of winglets (semi ellipses, triangles, and rectangles, etc.), planar contour (number of slots, length, and width of wingtip slots), and spatial pattern, i.e., horizontal and vertical distribution parameters (angle of attack, twist angle, sweep angle, and cant angle of winglets) can be studied independently, to analyze the function of each parameter. The authors emphasize that the aspect ratio should be consistent as far as possible in modeling, otherwise the conclusions need to be further discussed.

The choice of numerical simulation method depends on the expectation and computing resources to be spent of researchers [111, 112]. In this instance, Reynolds averaged $\mathrm{N}-\mathrm{S}$ equation method (RANS) can be utilized to quickly get
Table 2 Impact parameters of aerodynamic properties on wingtip slots

\begin{tabular}{llll}
\hline Structure parameters & Cant angle & Twist angle & Sweep angle \\
& Number of slots & Width of slots & Length of slots \\
& Shape of winglets & Angle of attack for winglets & Fold \\
Motion parameters & Plunge & Twist & Sweep \\
Deformation parameters & Chordwise flexibility & Spanwise flexibility & \\
\hline
\end{tabular}




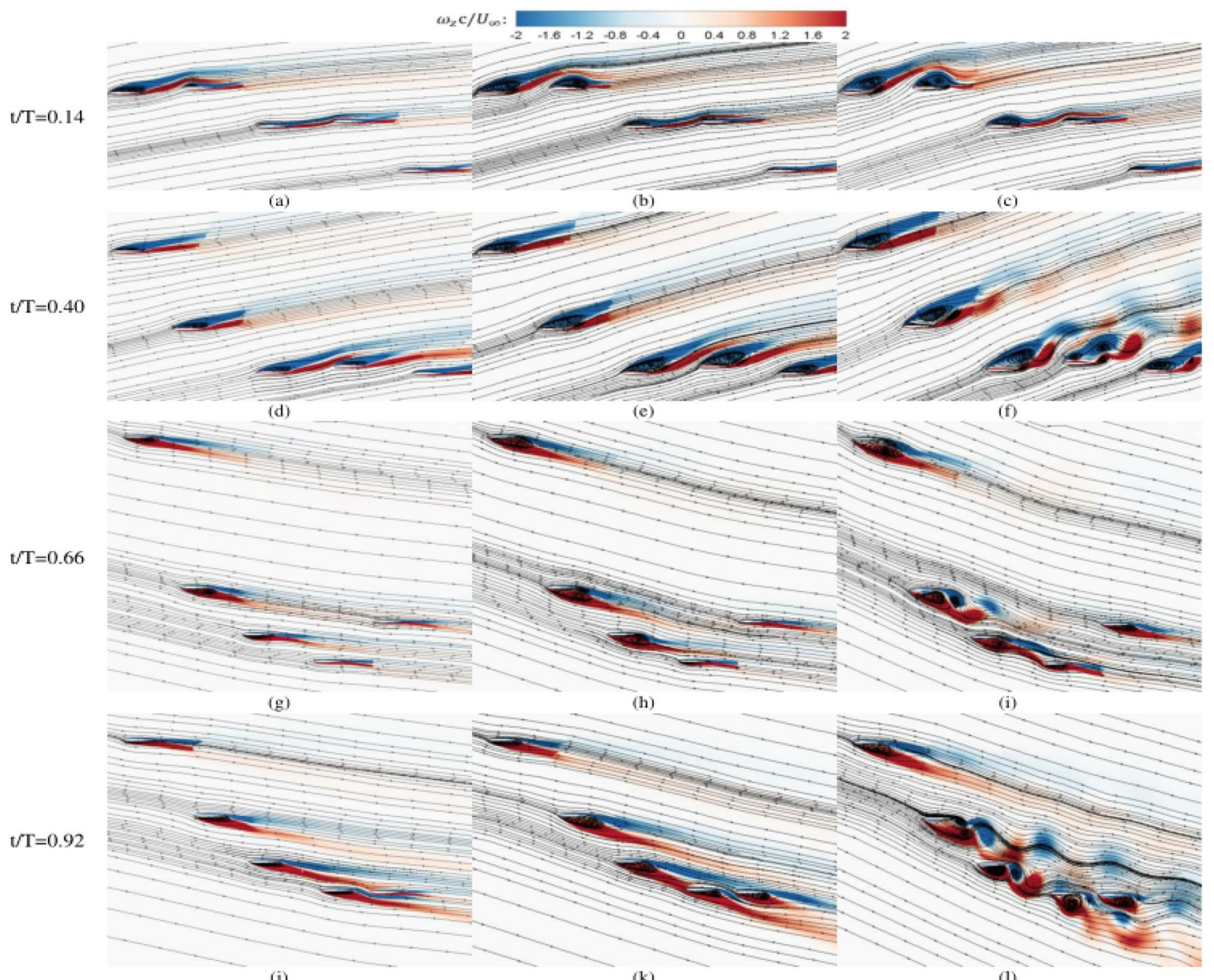

(j)

(k)

(1)

Fig. 40 Instantaneous streamlines and z-vorticity contours of slotted wing at the instant of (a,b,c) $t / T=0.14,(\mathrm{~d}, \mathrm{e}, \mathrm{f}) t / T=0.40,(\mathrm{~g}, \mathrm{~h}, \mathrm{i}) t / T=0.66$, and $(\mathrm{j}, \mathrm{k}, \mathrm{l}) t / T=0.92$ for flapping frequency $f=1.14 \mathrm{~Hz}, f=1.71 \mathrm{~Hz}$ and $f=2.28 \mathrm{~Hz}[90]$

the regular results of aerodynamic characteristics changes of bionic slotted wings under the influence of different parameters [113, 114].

In recent years, two advanced methods of drag-decomposition based on momentum conservation theory have been widely concerned. One of the methods is called wake integral, or the far-field method, which can compute drag components from the surface integration on the wake plane [115]. This method is not affected by the surface of the object, and it is also applicable to the complex shape. But like the surface integral method, the far-field method has higher requirements on the mesh amount [116]. The other method is called flow field integration, or the midfield method. Instead of crude force and moment comparison, flow field integration, derived from the far-field method by applying the divergence theorem, can compute drag components from the volume integration around the wings [117]. Flow field integration decomposes drag into induced drag, wave drag, profile drag, and spurious drag caused by numerical diffusion. Therefore, numerical dissipation drag can be separated to get more accurate results [118]. It has been proved that the flow field integration method is effective in the optimal design of airfoils, wings, and wingtips [119]. Another advantage of the midfield method is that it enables decompose and visualize drag [107]. What's more, physical drag components evaluated by the flow field integration method were almost independent of the mesh resolution [120]. Hence, the drag reduction mechanism can be well studied by the drag component obtained from flow field integration.

PIV technology has evolved to be the dominant method for velocimetry in experimental fluid mechanics, especially contributed to the study of flows in complex geometries (just like slotted wings) and turbulent flows, providing resolution and detail that can compete with 


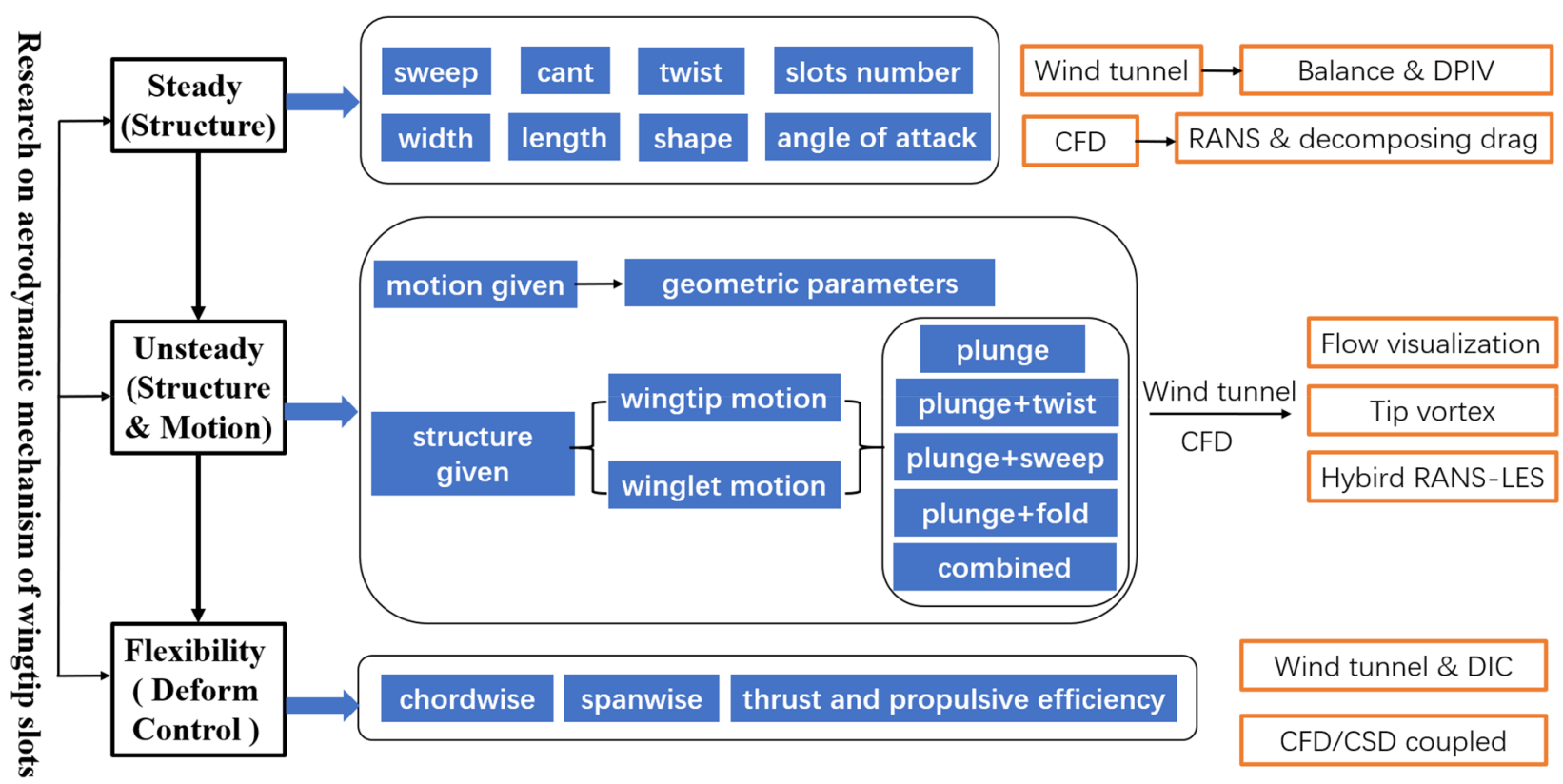

Fig. 41 Road map for future research on aerodynamic mechanism of wingtip slots

modern numerical methods, such as DNS (direct numerical simulation) [121]. Researchers can study the influence of assorted bionic slotted wingtip configurations on the aerodynamic performance of the wing and flow field properties around the wing with the aid of numerical simulation and wind tunnel experiment-assisted PIV technology.

\subsection{Geometric Parameters and Kinematic Parameters in Unsteady State}

The research of unsteady state can be carried out from two aspects, one is multiple geometric parameters investigation of wingtip slots under given flapping motion, and the other is various motion parameters study under given wingtip configuration. The former is basically the same as that of the steady state, and wing models with diverse control variables is also established for independent research under the condition that other parameters remain constant to remove unconcerned variable disturbance.

As for motion parameter study, in the respect of the wingtip is also an integral part of the wing, any deformation of the wings' kinematic motion like flapping, torsion, folding, forward and backward sweeping, will have an impact on the wingtips' effect. In addition, the structure of slotted wingtip also appears diverse shape in different flight stages, which is an adaptive adjustment process for a bird's flight state. Therefore, in addition to the study of the influence of an entire bionic slotted wingtip, the interaction between slotted winglets should be included as a part of the study as well.
To sum up, the research on flapping motion of wingtip slots can be divided into two categories, namely (a) evaluating the effect of the entire movement of wingtips and (b) exploring the movement mechanism among winglet structures under assorted kinematic modes.

(a) The whole movement of wingtip means that there is no relative position and motion change in the process of flapping among multiple winglets, where only the whole wingtip and the main wing are taken into account.

(b) When the relative movement between winglets is discussed, the slotted wingtip is no longer regarded as an entire part, their relative position and shape of each winglet are changed.

The flight of birds in nature is often formed by combining four typical kinematic modes of birds: plunging, twisting, sweeping, and folding. The impact of slotted wingtips with the following motion modes under varying Strouhal number and kinematic parameters (listed in parentheses) could be studied.

(1) Pure plunging motion (plunging amplitude, frequency)

(2) Plunging and twisting (plunging amplitude, twisting amplitude, frequency, phase difference)

(3) Plunging and sweeping (plunging amplitude, sweeping amplitude, frequency, phase difference)

(4) Plunging and folding (plunging amplitude, folding amplitude, frequency, phase difference)

(5) Plunging and twisting and sweeping and folding (amplitude, frequency, phase difference) 
After studying the influence of the parameters of the whole wingtip movement and relative movement among winglets under these five coupled movements, cooperated with the comparison of aerodynamic force coefficient and tip vortex spreading, the effects of the dynamic adjustment process of wingtip slots on aerodynamics, flight stability, and wingtip vortex evolutional mechanism are revealed.

Unlike the steady state, the unsteady flow has large-scale separation characteristics, especially near slotted tips. However, The RANS method gives the average motion results, which cannot reflect the detailed information of the turbulence $[122,123]$. It is not suitable for large separation flow, even complicated to meet the prediction accuracy of complex unsteady turbulence $[124,125]$. In view of rigid and complex flapping motion, the RANS-LES hybrid method is an ideal method to describe unsteady turbulent flow [126].

As wind tunnel tests are taken into account, the PIV technique is also suitable for the study of complex flow structures such as vortex and turbulence, especially for the display of the formation, development, and evolution of tip vortex of the slotted wing with multiple geometric and kinematic parameters [127].

\subsection{Flexibility of Slotted Wingtips}

Shyy et al. [95] comprehensively reviewed the research progress of flexible wings and pointed out that appropriate chordwise flexibility and spanwise flexibility can enhance thrust and propulsion efficiency. Therefore, it is necessary to consider the flexibility of slotted wingtips when exploring the influence of geometric parameters and kinematic parameters. The dynamic aerodynamic properties can be evaluated under the conditions that equipped wingtip slots or not, various wingtip configurations, and motion modes as previously described.

As flexibility is introduced into slotted wingtips, analysis of the effects of geometric and kinematic parameters on the flexible deformation and aerodynamic properties of wings is needed, while flexible wingtips' impact on the flow field and tip vortex structure ought to be evaluated.

The deformations of flexible wings affect features of the flow field, and the forces of the flow field also change deformations of flexible wings accordingly [128-130]. For such a strongly coupled, complex fluid-structure interaction (FSI) problem of the flapping wing can acquire more reasonable results with the aid of fluid-structure interaction analysis method than independent flow field simulation or structural analysis [131]. FSI can be divided into one-way FSI and two-way FSI. The former alters the structure by deformation, displacement, and vibration settings, thus influence the fluid. The latter takes into account the structural variation caused by aerodynamic force and the aerodynamic characteristic change owing to the surface structure change caused by flow itself [132]. The computational FSI analysis by coupling CFD solver and computational structural dynamics (CSD) solver is a direct and effective method to resolve this highly nonlinear problem. It can provide detailed information of flow field and structural dynamics of the flexible wing, to evaluate the aerodynamic behavior of the flexible wing [133, 134].

For solving the fluid-structure interaction problem of flexible wingtip at high Reynolds numbers, similar to the FSI problem of conventional wings, how to measure and simulate small deformations and how to couple fluid and structural modules become major obstacles due to the imperfection of experimental methods and numerical simulation tools. We reckon the flexibility deformation can be modeled by the image data of a high-speed camera, and structural modeling is carried out according to the primary feather material of birds. Then they are coupled with CFD for calculation. For wind tunnel experiments, the deformation measurement technique based on a high-speed camera system can be applied to measure the flexible deformation of slotted wingtips [135]. In this method, the spatial coordinates of experimental models are calculated by Digital Image Correlation (DIC) method, and the local deformation is obtained by data processing [136]. PIV is used for flow measurement and provides instantaneous velocity fields over global domains, can accurately obtain deformation of wings and its impact on flight performance [137, 138].

At present, the mainstream method to solve the FSI problem is pure numerical simulation. For example, the current flutter analysis is largely solved by numerical simulations. The solutions of the structural module and fluid module are obtained by solving equations. There are also many pure numerical FSI about flapping wings [139]. The difficulty of pure numerical FSI mainly lies in how to realize highprecision bi-directional coupling and the huge amount of calculation brought by high-precision requirements.

\section{Conclusions}

This paper reviews the research status of the aerodynamic mechanism of the wingtip slots and attempts to clarify some main research directions and strategies for future study. The following conclusions can be drawn:

(1) Previous studies focused on the parameters of wingtip slots under steady state, the majority of scholars conducted their research in the aid of wind tunnel tests and received wingtip slots effect on lift improvement, drag reduction, and yaw stabilization. However, there are still advancements and shortcomings in the previous research on wingtip slots. 
(2) In the aspect of research contents, besides geometric parameters in steady state, it is suggested that unsteady state of flapping and flexibility of wing should be considered. The unsteady study can be carried out from two aspects, one is multiple geometric parameters investigation of wingtip slots under given flapping motion, and the other is various motion parameters consideration under given wingtip configuration. In addition to the influence of an entire slotted wingtip, the interaction between slotted winglets should be included as a part of the study as well.

(3) As for research strategies, wind tunnel tests and numerical simulation methods should be applied comprehensively from the aspects of expected results and time cost, of course, both methods can be carried out synchronously if necessary. It should be noted that the numerical simulation method listed in this paper is not unique, if computing resources and time are sufficient, researchers can use the more accurate methods like LES (Large eddy simulation) and DNS (Direct numerical simulation). It is inaccurate to judge the role of the wingtip slots only according to the change of aerodynamic force of a whole wing to a certain extent. More accurate strategies, like drag decomposition and flow field visualization, are recommended.

Acknowledgements The authors would like to express their thanks for the support from National Natural Science Foundation of China (Grant 11872314 and U1613227), Youth Program of Natural Science Basic Research Plan in Shaanxi Province of China (Grant 2019JQ-394) and Key R\&D Program in Shaanxi Province of China (Grant 2020GY-154).

Funding The research leading to these results received funding from National Natural Science Foundation of China (Grant 11872314 and U1613227), Youth Program of Natural Science Basic Research Plan in Shaanxi Province of China (Grant 2019JQ-394) and Key R\&D Program in Shaanxi Province of China (Grant 2020GY-154).

\section{Declarations}

Conflict of interest The authors declare that they have no known competing financial interests or personal relationshipsthat could have appeared to influence the work reported in this paper. The authors have no competing interests to declare that are relevant to the content of this article.

Open Access This article is licensed under a Creative Commons Attribution 4.0 International License, which permits use, sharing, adaptation, distribution and reproduction in any medium or format, as long as you give appropriate credit to the original author(s) and the source, provide a link to the Creative Commons licence, and indicate if changes were made. The images or other third party material in this article are included in the article's Creative Commons licence, unless indicated otherwise in a credit line to the material. If material is not included in the article's Creative Commons licence and your intended use is not permitted by statutory regulation or exceeds the permitted use, you will need to obtain permission directly from the copyright holder. To view a copy of this licence, visit http://creativecommons.org/licenses/by/4.0/.

\section{References}

1. Lockwood, R., Swaddle, J. P., \& Rayner, J. M. V. (1998). Avian wingtip shape reconsidered: wingtip shape indices and morphological adaptations to migration. Journal of Avian Biology, 29, 273-292.

2. Shyy, W., Berg, M., \& Ljungqvist, D. (1999). Flapping and flexible wings for biological and micro air vehicles. Progress in Aerospace Science, 35, 455-505.

3. Feo, T. J., Field, D. J., \& Prum, R. O. (2015). Barb geometry of asymmetrical feathers reveals a transitional morphology in the evolution of avian flight. Proceedings of the Royal Society B: Biological Sciences, 282, 20142864.

4. Mohamed, A., Massey, K., Watkins, S., \& Clothier, R. (2014). The attitude control of fixed-wing MAVS in turbulent environments. Progress in Aerospace Sciences, 66, 37-48.

5. Withers, P. C. (1981). An aerodynamic analysis of bird wings as fixed aerofoils. Journal of Experimental Biology, 90, 143-162.

6. Försching, H., \& Hennings, H. (2012). Aeroelastic mysteries in avian flight. CEAS Aeronautical Journal, 3, 135-143.

7. Burton, R. (1991). Vogelflug - Aerodynamik, Anatomie. Anpassung. Franckh-Kosmos Verlag.

8. Savile, D. B. O. (1957). Adaptive evolution in the avian wing. Evolution, 11, 212-224.

9. Shyamal, L. Counteracting extremes enabling normal flying: insights for global governance from birds on the wing and the dodo, [2019-12-05], https://www.laetusinpraesens.org/docs10s/ flying.php

10. Mueller, T. J. (1999). Aerodynamic measurements at low Reynolds numbers for fixed wing micro-air vehicles (pp. 1-32). Notre dame univ in dept of aerospace and mechanical engineering.

11. Nakata, T., Liu, H., Tanaka, Y., Nishihashi, N., Wang, X., \& Sato, A. (2011). Aerodynamics of a bio-inspired flexible flapping-wing micro air vehicle. Bioinspiration \& Biomimetics, 6, 045002.

12. Beaufrere, H. (2009). A review of biomechanic and aerodynamic considerations of the avian thoracic limb. Journal of Avian Medicine and Surgery, 23, 173-185.

13. Kim, D., Lee, S. H., \& Kim, D. (2019). Aerodynamic interaction of collective plates in side-by-side arrangement. Physics of Fluids, 31, 071902.

14. Tobalske, B. W. (2000). Biomechanics and physiology of gait selection in flying birds. Physiological and Biochemical Zoology, $73,736-750$

15. Aldheeb, M. A., Asrar, W., Sulaeman, E., \& Omar, A. A. (2016). A review on aerodynamics of non-flapping bird wings. Journal of Aerospace Technology and Management, 8, 7-17.

16. Guerrero, J. E., Maestro, D., \& Bottaro, A. (2012). Biomimetic spiroid winglets for lift and drag control. Comptes Rendus Mecanique, 340, 67-80.

17. Withers, P. C. (1981). The aerodynamic performance of the wing in red-shouldered hawk buteo lznearzs and a possible aeroelastic role of wing-tip slots. Ibis, 123, 239-247.

18. Tucker, V. A. (1995). Drag reduction by wing tip slots in a gliding Harris' hawk, Parabuteo unicinctus. Journal of Experimental Biology, 198, 775-781.

19. March, A. I., Bradley, C. W., \& Garcia, E. (2005, January). Aerodynamic properties of avian flight as a function of wing shape. In ASME International Mechanical Engineering Congress and Exposition (Vol. 42193, pp. 955-963)

20. Kleinheerenbrink, M., Johansson, L. C., \& Hedenström, A. (2017). Multi-cored vortices support function of slotted wing 
tips of birds in gliding and flapping flight. Journal of the Royal Society Interface, 14, 20170099.

21. KleinHeerenbrink, M., Warfvinge, K., \& Hedenström, A. (2016). Wake analysis of aerodynamic components for the glide envelope of a jackdaw (Corvus monedula). Journal of Experimental Biology, 219, 1572-1581.

22. Nadal, J., Ponz, C., \& Margalida, A. (2018). Feathers for escape: the transition from juvenile to adult in red-legged partridges (Alectoris rufa). Biological Journal of the Linnean Society, 123, 72-80.

23. Crandell, K. E., \& Tobalske, B. W. (2011). Aerodynamics of tipreversal upstroke in a revolving pigeon wing. Journal of Experimental Biology, 214, 1867-1873.

24. Sachs, G., \& Moelyadi, M. A. (2006). Effect of slotted wing tips on yawing moment characteristics. Journal of Theoretical Biology, 239, 93-100.

25. Saiteja, A., \& Suresh, C. (2014). Computational analysis of slotted wing tips at low Reynolds number. International Journal of Scientific \& Technology Research, 3, 360-363.

26. Fluck, M., \& Crawford, C. (2014). A lifting line model to investigate the influence of tip feathers on wing performance. Bioinspiration \& Biomimetics, 9, 046017.

27. Tucker, V. A. (1993). Gliding birds: reduction of induced drag by wing tip slots between the primary feathers. Journal of Experimental Biology, 180, 285-310.

28. Hossain, A., Rahman, A., Iqbal, A., Ariffin, M., \& Mazian, M. (2012). Drag analysis of an aircraft wing model with and without bird feather like winglet. International Journal of Aerospace and Mechanical Engineering, 6, 8-13.

29. Mitchell, T., \& Jacob, J. (2013). Evaluation of Bird-WingInspired Features on Biomimetic UAVs. In 51st AIAA Aerospace Sciences Meeting including the New Horizons Forum and Aerospace Exposition (p. 89).

30. Smith, M., Komerath, N., Ames, R., Wong, O., \& Pearson, J. (2001). Performance analysis of a wing with multiple winglets. In 19th AIAA Applied Aerodynamics Conference (p. 2407)

31. Yang, W. Q., \& Song, B. F. (2017). Experimental investigation of aerodynamics of feather-covered flapping wing. Applied Bionics and Biomechanics, 2017, 3019640.

32. Siddiqui, N. A., Aldeeb, M., Asrar, W., \& Sulaeman, E. (2018). Experimental investigation of a new spiral wingtip. International Journal of Aviation, Aeronautics, and Aerospace, 5, 6.

33. Lynch, M., Mandadzhiev, B., \& Wissa, A. (2018). Bioinspired wingtip devices: a pathway to improve aerodynamic performance during low Reynolds number flight. Bioinspiration \& Biomimetics, 13, 036003

34. Hui, Z., Cheng, G., \& Chen, G. (2021). Experimental investigation on tip-vortex flow characteristics of novel bionic multi-tip winglet configurations. Physics of Fluids, 33, 011902.

35. Coiro, D. P., Nicolosi, F., Scherillo, F., \& Maisto, U. (2008). Single versus multiple winglets: numerical and experimental investigation. In 26th International Congress of the Aeronautical Sciences, Anchorage (pp. 1-11)

36. Coiro, D. P., Nicolosi, F., Scherillo, F., \& Maisto, U. (2008). Improving hang-glider maneuverability using multiple winglets: a numerical and experimental investigation. Journal of Aircraft, 45, 981-989.

37. Coiro, D. P., Nicolosi, F., Scherillo, F., \& Maisto, U. (2008). Design of multiple winglets to improve turning and soaring characteristics of Angelo D'Arrigo's hang-glider: Numerical and experimental investigation. Aerotecnica Missili \& Spazio, $87,74-85$.

38. La Roche, U., \& Palffy, S. (1996, September). Wing-grid, a novel device for reduction of induced drag on wings. In ICAS PROCEEDINGS (Vol. 20, pp. 2303-2309)
39. Brothers E. Airbus unveils hybrid-electric regional airliner concept, [2019-07-22], http://www.avi-ation.cn/2019/0720/54787. html

40. Spillman, J. J. (1978). The use of wing tip sails to reduce vortex drag. Aeronautical Journal, 82, 387-395.

41. Parkin, C. S., \& Spillman, J. J. (1980). The use of wing-tip sails on a spraying aircraft to reduce the amount of material carried off-target by a crosswind. Journal of Agricultural Engineering Research, 25, 65-74.

42. Spillman, J. J., \& McVitie, A. M. (1984). Wing tip sails which give lower drag at all normal flight speeds. Aeronautical Journal, $88,362-369$

43. Spillman, J. J. (1987). Wing tip sails; progress to date and future developments. Aeronautical Journal, 91, 445-453.

44. Zimmer, H. (1987). The aerodynamic optimization of wings at subsonic speeds and the influence of wingtip design. National Aeronautics and Space Administration. NASA TM-88534

45. Chen, M. Y., \& Qi, M. B. (1994). The research of increased lift and reduced drag for wing-tip sails. Acta Aeronautica et Astronautica Sinica, 15, 641-646.

46. Qi, M. B., \& Chen, M. Y. (1995). The study of flow field near the wing tip and the mechanism of drag reduction for wing tip sails. Aerodynamic Experiment and Measurement \& Control, 9, $38-45$.

47. Cerón-Muñoz, H. D., \& Catalano, F. M. (2006). Experimental analysis of the aerodynamic characteristics adaptive of multiwinglets. Proceedings of the Institution of Mechanical Engineers, Part G: Journal of Aerospace Engineering, 220, 209-215.

48. Cerón-Muñoz, H. D., Cosin, R., Coimbra, R. F. F., Correa, L. G. N., \& Catalano, F. M. (2013). Experimental investigation of wing-tip devices on the reduction of induced drag. Journal of Aircraft, 50, 441-449.

49. Al-Atabi, M. (2006). Aerodynamics of wing tip sails. Journal of Engineering Science and Technology, 1, 89-98.

50. Chin, J., Al-Atabi, M. (2013). Investigation of wing tip sails. 1st Engineering Undergraduate Research Catalyst Conference (pp. 25-26)

51. Sun, C. H., \& Dai, C. (2015). Experimental study on ground effect of a wing with tip sails. Procedia Engineering, 126, 559-563.

52. Zhou, J. X., \& Sun, C. H. (2020). Drag reduction and flow structures of wing tip sails in ground effect. Journal of Hydrodynamics, 32, 93-106.

53. Han, M. W., Rodrigue, H., Kim, H. I., Song, S. H., \& Ahn, S. H. (2016). Shape memory alloy/glass fiber woven composite for soft morphing winglets of unmanned aerial vehicles. Composite Structures, 140, 202-212.

54. Hui, Z., Zhang, Y., \& Chen, G. (2020). Tip-vortex flow characteristics investigation of a novel bird-like morphing discrete wing structure. Physics of Fluids, 32, 035112.

55. Di Luca, M., Mintchev, S., Heitz, G., Noca, F., \& Floreano, D. (2017). Bioinspired morphing wings for extended flight envelope and roll control of small drones. Interface Focus, 7, 20160092.

56. Shelton, A., Tomar, A., Prasad, J. V. R., Smith, M. J., \& Komerath, N. (2006). Active multiple winglets for improved unmannedaerial-vehicle performance. Journal of aircraft, 43, 110-116.

57. BionicSwift - Safe aerial acrobatics as a swarm, [2019-07-22], https://www.festo.com/group/en/cms/13787.htm

58. Mishra, N., Gupta, A. S., Dawar, J., Kumar, A., \& Mitra, S. (2018). Numerical and experimental study on performance enhancement of darrieus vertical axis wind turbine with wingtip devices. Journal of Energy Resources Technology, 140, 121201.

59. Kroo, I. (2001). Drag due to lift: concepts for prediction and reduction. Annual Review of Fluid Mechanics, 33, 587-617.

60. Yoon, D., Kohama, Y., Kikuchi, S., \& Kato, T. (2006). Improvement of aerodynamic performance of the aero-train by 
controlling wing-wing interaction using single-slotted flap. JSME International Journal Series B Fluids and Thermal Engineering, $49,1118-1124$.

61. Barrios, J. P. L. G. M., \& Herman, G. M. P. (2017). Reducing drag by modifying the winglet design (pp. 1-29). The Philippine Physics Society Physics Fair.

62. Bannasch, R. (2001). From soaring and flapping bird flight to innovative wing and propeller constructions. Fixed and Flapping Wing Aerodynamics for Micro Air Vehicle Applications, 195, 453-472.

63. Whitcomb, R. T. (1976). A design approach and selected wind tunnel results at high subsonic speeds for wing-tip mounted winglets. NASA TN D-8260

64. Wauters, J., Couckuyt, I., Knudde, N., Dhaene, T., \& Degroote, J. (2020). Multi-objective optimization of a wing fence on an unmanned aerial vehicle using surrogate-derived gradients. Structural and Multidisciplinary Optimization, 61, 353-364.

65. Setyo Hariyadi S, P., Sutardi, W., \& Aries, W. (2018, August). Drag reduction analysis of wing airfoil E562 with forward wingtip fence at cant angle variations of $75^{\circ}$ and $90^{\circ}$. In American Institute of Physics Conference Series (Vol. 2001, No. 1, p. 050003)

66. Guerrero, J., Sanguineti, M., \& Wittkowski, K. (2018). CFD study of the impact of variable cant angle winglets on total drag reduction. Aerospace, 5, 126.

67. Beechook, A., \& Wang, J. (2013, September). Aerodynamic analysis of variable cant angle winglets for improved aircraft performance. In 2013 19th International Conference on Automation and Computing (pp. 1-6). IEEE

68. Munshi, A., Sulaeman, E., Omar, N., \& Ali, M. Y. (2018). CFD analysis on the effect of winglet cant angle on aerodynamics of ONERA M6 wing. Journal of Advanced Research in Fluid Mechanics and Thermal Sciences, 45, 44-54.

69. Gu, R. P., Song, G. P., Diao, H. Z., \& Liu, W. (2015). Numerical simulations of the civil aviation aircraft wings with or without wingtip vortex diffuser. Flight Dynamics, 33, 403-406. (in Chinese).

70. Puttinger, S., Mehrle, A., Gittler, P., \& Meile, W. (2011). Numerical optimization and experimental investigations on the principle of the vortex diffuser. Journal of Aircraft, 48, 845-854.

71. Sabet, G. S., Pavithran, S., \& Kulkarni, R. R. (2018). Comparison of heat transfer enhancement in a diffuser by inserting wing vortex generators and protrusions. Iranian Journal of Science and Technology, Transactions of Mechanical Engineering, 42, 213-220.

72. Blake, W. B., \& Johnson, A. F. (2009). Assessment of straight tapered geometry approximations of raked-tip wings. Journal of Spacecraft and Rockets, 46, 103-111.

73. Swanstrom, F. M., \& Hawke, T. (2000). Design for manufacturing and assembly: a case study in cost reduction for composite wing tip structures. SAMPE Journal, 36, 9-16.

74. Ahluwalia, S. K., Negi, V., Anand, N., Goyal, V., \& Singh, R. K. (2017). CFD analysis on different shapes of winglet at low subsonic flow. International Journal of Advance Research and Innovation, 5, 446-451.

75. Mahmood, C. A., \& Das, R. K. (2019). Performance comparison of different winglets by CFD. In AIP Conference Proceedings (Vol. 2121, No. 1, p. 060006)

76. Wan T, Chou H C, Lien K W. Aerodynamic efficiency study of modern spiroid winglets. 25th Congress of International Council of the Aeronautical, Sciences, Hamburg, Germany, 2006, ICAS 2006-3.7S

77. Tabata, S., Yamazaki, W., \& Yuhara, T. (2020). Drag/Weight reduction using split-tip winglet for TRA2012A model. Transactions of the Japan Society for Aeronautical and Space Sciences, $63,69-79$.
78. Gunasekaran, S., \& Gerham, T. (2018). Effect of chordwise slots on aerodynamic efficiency and wingtip vortex. AIAA Journal, 56, $4752-4767$.

79. Deslich, J., \& Gunasekaran, S. (2019). Effect of Slotted Winglet on the Wingtip Vortex. In AIAA Aviation 2019 Forum (p. 3431)

80. Boling, J. S., Zha, G., \& Altman, A. (2020). Numerical Investigation of Wingtip Vortices of Coflow Jet Active Flow Control Wings. In AIAA AVIATION 2020 FORUM (p. 2943)

81. García-Ortiz, J. H., Blanco-Rodríguez, F. J., Parras, L., \& Del Pino, C. (2020). Experimental observations of the effects of spanwise blowing on the wingtip vortex evolution at low Reynolds numbers. European Journal of Mechanics-B/Fluids, 80, 133-145.

82. Asadpour, M., Van den Bergh, B., Giustiniano, D., Hummel, K. A., Pollin, S., \& Plattner, B. (2014). Micro aerial vehicle networks: an experimental analysis of challenges and opportunities. IEEE Communications Magazine, 52, 141-149.

83. Newman, B. G. (1958). Soaring and gliding flight of the black vulture. Journal of Experimental Biology, 35, 280-285.

84. Khan, F. N., Batul, B., \& Aizaz, A. (2019, October). A CFD Analysis of Wingtip Devices to Improve Lift and Drag Characteristics of Aircraft Wing. In IOP Conference Series: Materials Science and Engineering (Vol. 642, No. 1, p. 012006)

85. Prandtl, L., \& Betz, A. (Eds.). (2009). Ergebnisse der aerodynamischen Versuchsanstalt zu Göttingen (Vol. 3). Universitätsverlag Göttingen

86. Kroo, I. (2005). Nonplanar wing concepts for increased aircraft efficiency. VKI lecture series on innovative configurations and advanced concepts for future civil aircraft. (pp. 1-29)

87. Hummel, D. (1978, September). Recent aerodynamic contributions to problems of bird flight. In ICAS Proceedings, 11th Congress of the International Council of the Aeronautical Sciences (pp. 10-16)

88. Tay, W. B. (2017). Effect of different types of wing-wing interactions in flapping MAVs. Journal of Bionic Engineering, 14, 60-74.

89. Sane, S. P., \& Dickinson, M. H. (2002). The aerodynamic effects of wing rotation and a revised quasi-steady model of flapping flight. Journal of Experimental Biology, 205, 1087-1096.

90. Liu, D., Song, B. F., Yang, W. Q., Xue, D., \& Lang, X. Y. (2021). Unsteady characteristic research on aerodynamic interaction of slotted wingtip in flapping kinematics. Chinese Journal of Aeronautics. https://doi.org/10.1016/j.cja.2021.07.010

91. Kang, C. K., Aono, H., Cesnik, C. E. S., \& Shyy, W. (2011). Effects of flexibility on the aerodynamic performance of flapping wings. Journal of Fluid Mechanics, 689, 32-74.

92. Young, J., Walker, S. M., Bomphrey, R. J., Taylor, G. K., \& Thomas, A. L. R. (2009). Details of insect wing design and deformation enhance aerodynamic function and flight efficiency. Science, 325, 1549-1552.

93. Heathcote, S., Wang, Z., \& Gursul, I. (2008). Effect of spanwise flexibility on flapping wing propulsion. Journal of Fluids and Structures, 24, 183-199.

94. Shyy, W., Lian, Y., Tang, J., Viieru, D., \& Liu, H. (2007). Aerodynamics of Low Reynolds Number Flyers. Cambridge University Press.

95. Shyy, W., Aono, H., Chimakurthi, S. K., Trizila, P., Kang, C. K., Cesnik, C. E., \& Liu, H. (2010). Recent progress in flapping wing aerodynamics and aeroelasticity. Progress in Aerospace Sciences, 46, 284-327.

96. Roe, P. L. (1986). Characteristic-based schemes for the Euler equations. Annual Review of Fluid Mechanics, 18, 337-365.

97. Kato, T., \& Ponce, G. (1988). Commutator estimates and the Euler and Navier-Stokes equations. Communications on Pure and Applied Mathematics, 41, 891-907. 
98. Shakib, F., Hughes, T. J. R., \& Johan, Z. (1991). A new finite element formulation for computational fluid dynamics: X. The compressible Euler and Navier-Stokes equations. Computer Methods in Applied Mechanics and Engineering, 89, 141-219.

99. Chorin, A. J. (1968). Numerical solution of the Navier-Stokes equations. Mathematics of Computation, 22, 745-762.

100. Ruelle, D., \& Takens, F. (1971). On the nature of turbulence. Communications in Mathematical Physics, 20, 167-192.

101. Pandit, R., Perlekar, P., \& Ray, S. S. (2009). Statistical properties of turbulence: an overview. Pramana, 73, 157-191.

102. Ecke, R. (2005). The turbulence problem: an experimentalist's perspective. Los Alamos Science, 29, 124-141.

103. Ho, S., Nassef, H., Pornsinsirirak, N., Tai, Y. C., \& Ho, C. M. (2003). Unsteady aerodynamics and flow control for flapping wing flyers. Progress in Aerospace Sciences, 39, 635-681.

104. Chao, D. D., \& van Dam, C. P. (1999). Airfoil drag prediction and decomposition. Journal of Aircraft, 36, 675-681.

105. Mele, B., Ostieri, M., \& Tognaccini, R. (2017). Aircraft lift and drag decomposition in transonic flows. Journal of Aircraft, 54, 1933-1944.

106. Yamazaki, W., Matsushima, K., \& Nakahashi, K. (2007). Drag decomposition-based adaptive mesh refinement. Journal of Aircraft, 44, 1896-1905.

107. Gariepy, M., Trepanier, J. Y., \& Malouin, B. (2013). Generalization of the far-field drag decomposition method to unsteady flows. AIAA Journal, 51, 1309-1319.

108. Janus, J. M., \& Chatterjee, A. (1996). Use of a wake-integral method for computational drag analysis. AIAA Journal, 34, 188-190.

109. Kusunose, K. (1998). Drag prediction based on a wake-integral method. In 16th AIAA Applied Aerodynamics Conference (p. 2723)

110. Van Truong, T., Le, T. Q., Tran, H. T., Park, H. C., Yoon, K. J., \& Byun, D. (2012). Flow visualization of rhinoceros beetle (Trypoxylus dichotomus) in free flight. Journal of Bionic Engineering, 9, 304-314.

111. Spalart, P. R. (2000). Strategies for turbulence modelling and simulations. International Journal of Heat and Fluid Flow, 21, 252-263.

112. Kajishima, T., \& Taira, K. (2017). Numerical simulation of turbulent flows (pp. 207-235). Springer.

113. Lehnhauser, T., Ertem-Muller, S., Schafer, M., \& Janicka, J. (2004). Advances in numerical methods for simulating turbulent flows. Progress in Computational Fluid Dynamics, 4, 208-228.

114. Qu, F., \& Sun, D. (2017). Investigation into the influences of the low-speed flows' accuracy on RANS simulations. Aerospace Science and Technology, 70, 578-589.

115. Fan, Y. T., \& Li, W. P. (2019). Review of far-field drag decomposition methods for aircraft design. Journal of Aircraft, 56, 11-21.

116. Hunt, D. L., Cummings, R. M., \& Giles, M. B. (1999). Wake integration for three-dimensional flow field computations: applications. Journal of Aircraft, 36, 366-373.

117. Cummings, R., Giles, M., \& Shrinivas, G. (1996). Analysis of the elements of drag in three-dimensional viscous and inviscid flows. In 14th applied aerodynamics conference (p. 2482)

118. Yamazaki, W., Matsushima, K., \& Nakahashi, K. (2008). Aerodynamic design optimization using the drag-decomposition method. AIAA Journal, 46, 1096-1106.

119. Cheung, S. H., Edwards, T. A., \& Lawrence, S. L. (1992). Application of computational fluid dynamics to sonic boom near- and mid-field prediction. Journal of Aircraft, 29, 920-926.
120. Paparone, L., \& Tognaccin, R. (2003). Computational fluid dynamics-based drag prediction and decomposition. AIAA Journal, 41, 1647-1657.

121. Winzen, A., Roidl, B., Klän, S., Klaas, M., \& Schröder, W. (2014). Particle-image velocimetry and force measurements of leading-edge serrations on owl-based wing models. Journal of Bionic Engineering, 11, 423-438.

122. Fröhlich, J., \& Von Terzi, D. (2008). Hybrid LES/RANS methods for the simulation of turbulent flows. Progress in Aerospace Sciences, 44, 349-377.

123. Chaouat, B. (2017). The state of the art of hybrid RANS/LES modeling for the simulation of turbulent flows. Flow, Turbulence and Combustion, 99, 279-327.

124. Spalart, P. R. (2009). Detached-eddy simulation. Annual Review of Fluid Mechanics, 41, 181-202.

125. Walters, D. K., Bhushan, S., Alam, M. F., \& Thompson, D. S. (2013). Investigation of a dynamic hybrid RANS/LES modelling methodology for finite-volume CFD simulations. Flow, Turbulence and Combustion, 91, 643-667.

126. Kolomenskiy, D., Paoli, R., Boussuge, J. F. (2014). Hybrid RANS-LES simulation of wingtip vortex dynamics. 4th ASME Joint US-European Fluids Engineering Division Summer Meeting, Chicago, USA, FEDSM2014-21349

127. Decaix, J., Müller, A., Favrel, A., Avellan, F., \& Münch, C. (2018). U-RANS Simulations and PIV Measurements of a Selfexcited Cavitation Vortex Rope in a Francis Turbine. In Advances in Hydroinformatics. SimHydro Conference pp. 1069-1083)

128. Heathcote, S., Martin, D., \& Gursul, I. (2004). Flexible flapping airfoil propulsion at zero freestream velocity. AIAA Journal, 42, 2196-2204.

129. Fu, J., Liu, X., Shyy, W., \& Qiu, H. (2018). Effects of flexibility and aspect ratio on the aerodynamic performance of flapping wings. Bioinspiration \& Biomimetics, 13, 036001.

130. Gong, C. L., Han, J. K., Yuan, Z. J., Fang, Z., \& Chen, G. (2019). Numerical investigation of the effects of different parameters on the thrust performance of three-dimensional flapping wings. Aerospace Science and Technology, 84, 431-445.

131. Shyy, W., Lian, Y., Tang, J., Liu, H., Trizila, P., Stanford, B., Bernal, L., Cesnik, C., Friedmann, P., \& Ifju, P. (2008). Computational aerodynamics of low Reynolds number plunging, pitching and flexible wings for MAV applications. Acta Mechanica Sinica, 24, 351-373.

132. Zhao, L., Huang, Q. F., Deng, X. Y., \& Sane, S. P. (2010). Aerodynamic effects of flexibility in flapping wings. Journal of the Royal Society Interface, 7, 485-497.

133. Hou, G. N., Wang, J., \& Layton, A. (2012). Numerical methods for fluid-structure interaction-a review. Communications in Computational Physics, 12, 337-377.

134. Thielicke, W., \& Stamhuis, E. J. (2015). The influence of wing morphology on the three-dimensional flow patterns of a flapping wing at bird scale. Journal of Fluid Mechanics, 768, 240-260.

135. Tay, W. B., Jadhav, S., \& Wang, J. L. (2020). Application and improvements of the wing deformation capture with simulation for flapping micro aerial vehicle. Journal of Bionic Engineering, 17, 1096-1108.

136. Westerweel, J. (1997). Fundamentals of digital particle image velocimetry. Measurement Science and Technology, 8, 1379-1392.

137. Adrian, R. J. (1991). Particle-imaging techniques for experimental fluid mechanics. Annual Review of Fluid Mechanics, 23, 261-304. 
138. Westerweel, J., Elsinga, G. E., \& Adrian, R. J. (2013). Particle image velocimetry for complex and turbulent flows. Annual Review of Fluid Mechanics, 45, 409-436.

139. Pfeiffer, A. T., Lee, J. S., Han, J. H., \& Baier, H. (2010). Erratum to: Ornithopter flight simulation based on flexible multi-body dynamics. Journal of Bionic Engineering, 7, 102-111.
Publisher's Note Springer Nature remains neutral with regard to jurisdictional claims in published maps and institutional affiliations. 